\title{
Unsupervised Brain Tumor Segmentation Using Knowledge-Based and Fuzzy Techniques
}

\author{
Matthew C. Clark, Lawrence O. Hall, Dmitry B. Goldgof, Robert Velthuizen, ${ }^{1}$ F. \\ Reed Murtaugh, and Martin S. Silbiger ${ }^{1}$ \\ Department of Computer Science and Engineering \\ ${ }^{1}$ Department of Radiology \\ University of South Florida \\ Tampa, Fl. 33620 \\ hall@csee.usf.edu
}

\section{Introduction}

According to the Brain Tumor Society, approximately 100,000 people in the United States will be diagnosed with a primary or metastatic brain tumor within the next 12 months [16]. One of the primary diagnostic and treatment evaluation tools for brain tumors has been magnetic resonance (MR) imaging. MR imaging has become a widelyused method of high quality medical imaging, especially in brain imaging where MR's soft tissue contrast and non-invasiveness are clear advantages. MR images can also be used to track the size of a brain tumor as it responds (or doesn't) to treatment. A reliable method for segmenting tumor would clearly be a useful tool [32, 31, 57]. Currently, however, there is no method widely accepted in clinical practice for quantitating tumor volumes from MR images [38]. The Eastern Cooperative Oncology group [17] uses an approximation to tumor cross-sectional area in the single MR slice with the largest contiguous, well-defined tumor. These manual measurements, however, have shown poor reproducibility and tumor response criteria based on these manual estimations have shown poor correlation with quantitative 2D and 3D metrics [10].

Computer-based brain tumor segmentation has remained largely experimental work, with approaches including multi-spectral analysis $[50,51,55,54,29,19]$, edge detection $[21,12,45,22,60,59,2]$, neural networks [35, 30, 44], and knowledge-based techniques $[25,42,37,13,28]$. Our efforts in $[34,7,6]$ showed that a combination of knowledge-based techniques and multi-spectral analysis could effectively detect pathology and label normal transaxial slices. Most of these efforts, however, have either dealt with normal data sets, or with neuro-psychiatric disorders with MR distribution characteristics similar to normals [9].

Supervised pattern recognition methods have also exhibited problems with reproducibility, due to significant intra and inter-observer variance introduced over multiple trials of training example selection [9]. Furthermore, because supervision, such as the selection of training examples, can be time consuming and requires domain "expertise" to be effective, supervised methods are unsuitable for clinical use. These limitations suggest the need for a fully automatic method for tumor volume measurement, not only for tracking tumor response to therapy, but in planning future treatment as well $[32,31,57,10]$.

Here, we present a knowledge-based paradigm that combines fuzzy techniques, multispectral analysis, and image processing algorithms, to produce an unsupervised system capable of automatically (no human intervention on a per volume basis) segmenting and labeling complete glioblastoma-multiforme tumor volumes from transaxial MR images over a period of time during which the tumor is treated. Unlike most other efforts in 
segmenting brain pathology, this system has also been tested on a large number of unseen images with a fixed parameter (rule) set (built from a set of "training images") and quantitatively compared with "ground truth" images. This allows tumor response to therapy to be tracked over repeat scans and aid radiologists in planning subsequent treatment. More importantly, the system's unsupervised nature avoids the problems of observer variability found in supervised methods, providing complete reproducibility of results. Furthermore, observer-based training examples are not required, making the system suitable for clinical use.

Using knowledge gained during "pre-processing" (pathology detection) in [6,8], extra-cranial tissues (air, skin, fat, etc.) are first removed based on the segmentation created by a fuzzy c-means clustering algorithm [5, 23]. The remaining pixels (really voxels since they have thickness) form an intra-cranial mask. An expert system uses information from multi-spectral and local statistical analysis to first separate suspected tumor from the rest of the intra-cranial mask, then refine the segmentation into a final set of regions containing tumor. A rule-based expert system shell, CLIPS [46, 20], is used to organize the system. Low level modules for image processing and high level modules for image analysis are all written in $\mathrm{C}$ and called as actions from the right hand sides of the rules.

Each slice was classified as abnormal by systems described in $[6,8]$. Of the tumor types that are found in the brain, glioblastoma-multiformes (Grade IV Gliomas) are the focus of this work. This tumor type was addressed first because of its relative compactness and tendency to enhance well with paramagnetic substances, such as gadolinium. For the purposes of tumor volume tracking, segmentations from contiguous slices (within the same volume) are merged to calculate total tumor size in 3D. The tumor segmentation matches well with radiologist-verified "ground truth" images and is comparable to results generated by a supervised segmentation technique.

The remainder of the paper is divided into four sections. Section II. discusses the slices processed and gives a brief overview of the system. Section III. details the system's the major processing stages and the knowledge used at each stage. The last two sections present the experimental results, an analysis of them, and future directions for this work.

\section{Domain Background}

\section{A. Slices of Interest for the Study}

The system described here can process any transaxial slice [43, 47] intersecting the brain cerebrum, starting from an initial slice 7 to $8 \mathrm{~cm}$ from the top of the brain and moving up towards the top of the head and down towards the shoulders. This "initial slice," which simply needs to intersect the ventricles, is used as the starting point due to the relatively good signal uniformity within the MR coil used [6]. Each brain slice consists of three feature images: T1-weighted (T1), proton density weighted (PD), and T2-weighted (T2) [57] .

An example of a normal slice after processing is shown in Figures 1.1(a) and (b). Figures 1.1(c) and (d) show an abnormal slice through the ventricles, though pathology may exist within any given slice. The labeled normal intra-cranial tissues of interest are: CSF (dark gray) and the parenchymal tissues, white matter (white) and gray matter (black). In the abnormal slice, pathology (light gray) occupies an area that would otherwise belong to normal tissues. In the approach described here, only part of the pathology (gadolinium-enhanced tumor) is identified and labeled.

A total of 385 slices containing radiologist diagnosed glioblastoma-multiforme tumor were available for processing. Table 1..4 in Section IV. lists the distribution of these slices 


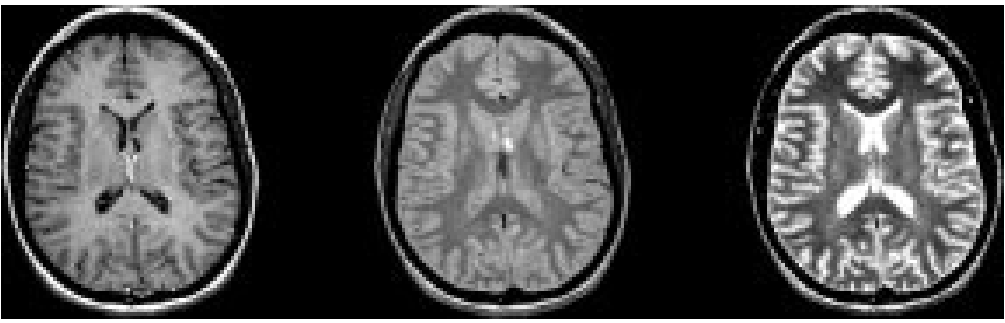

(a)

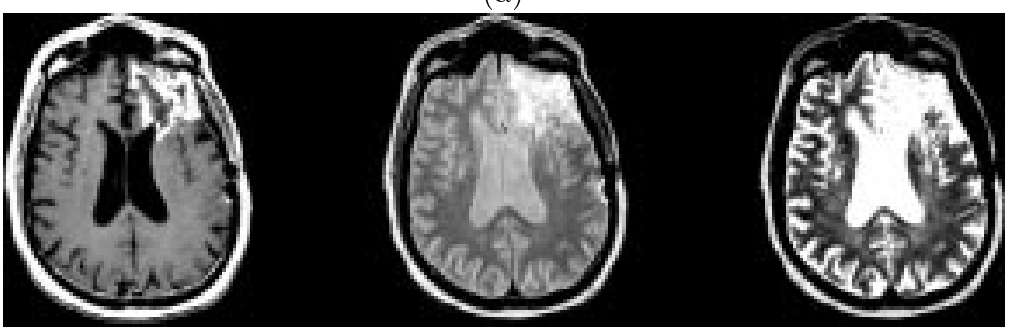

(c)

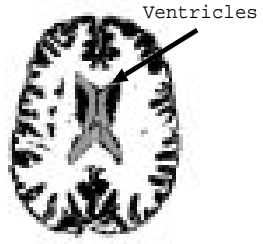

(b)

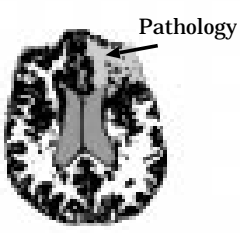

(d)

Figure 1.1: Slices of Interest: (a) raw data from a normal slice (T1-weighted, PD and T2-weighted images from left to right) (b) after segmentation (c) raw data from an abnormal slice (T1-weighted, PD and T2-weighted images from left to right) (d) after segmentation. White= white matter; Black=gray matter; Dark Gray=CSF; Light Gray= Pathology in (b) and (d).

across 33 volumes of eight patients who received varying levels of treatment, including surgery, radiation therapy, and chemo-therapy prior to initial acquisition and between subsequent acquisitions. Using a criteria of tumor size (per slice) and level of gadolinium enhancement to capture the required characteristics of all data sets acquired with this protocol, a training subset of 65 slices was created. The heuristics to be discussed in Section III. were manually extracted from the training subset through the process of "knowledge engineering" and are expressed in general terms, such as "higher end of the T1 spectrum" (which does not specify an actual T1 value). This provides knowledge that is more robust across slices, and avoids dependence on a slice's particular thickness, scanning protocol, or signal intensity, as was the case in [6].

\section{B. Basic MR Contrast Principles}

One of the key advantages in MR imaging is its ability to acquire multispectral data by rescanning a patient with different combinations of pulse sequence parameters (in our case repetition time (TR), echo time (TE)). For example, the MR data used in this study consists of T1, PD, and T2-weighted feature images. A T1-weighted image is produced by a relatively short TR/short TE sequence, a PD-weighted image uses a long TR/short TE sequence, while a long TR/long TE sequence produces a T2-weighted image [36, 15]. For the purpose of brevity, the T1-weighted, PD-weighted, and T2-weighted features will be referred to as $\mathrm{T} 1, \mathrm{PD}$, and $\mathrm{T} 2$ respectively.

A particular pulse sequence parameter will provide the best contrast between different tissue types [36] and a series of these images can be combined to provide a multispectral data set. The physics of these pulse sequences are outside the scope of this paper and their discussion is left to other literature sources [36, 15, 48]. We are primarily concerned with which pulse sequences best delineate specific tissues. After reviewing the available literature, a brief synopsis is shown in Table 1..1. This synopsis 
Table 1..1: A Synopsis of T1, PD, and T2 Effects on the Magnetic Resonance Image. $\mathrm{TR}=$ Repetition Time; $\mathrm{TE}=$ Echo Time.

\begin{tabular}{|c|c|c|}
\hline $\begin{array}{c}\text { Pulse Sequence } \\
\text { (TR/TE) }\end{array}$ & $\begin{array}{c}\text { Effect } \\
\text { (Signal Intensity) }\end{array}$ & Tissues \\
\hline \hline $\begin{array}{c}\text { T1-weighted } \\
\text { (short/short) }\end{array}$ & $\begin{array}{c}\text { Short T1 relaxation } \\
\text { (bright) }\end{array}$ & $\begin{array}{c}\text { Fat, Proteinaceous Fluid, } \\
\text { Paramagnetic Substances (Gadolinium) }\end{array}$ \\
\cline { 2 - 3 } & $\begin{array}{c}\text { Long T1 relaxation } \\
\text { (dark) }\end{array}$ & $\begin{array}{c}\text { Neoplasms, Edema, CSF, } \\
\text { Pure Fluid, Inflammation }\end{array}$ \\
\hline $\begin{array}{c}\text { PD-weighted } \\
\text { (long/short) }\end{array}$ & $\begin{array}{c}\text { High proton density } \\
\text { (bright) }\end{array}$ & Fat, Blood \\
\cline { 2 - 3 } & $\begin{array}{c}\text { Low proton density } \\
\text { (dark) }\end{array}$ & Fluids, CSF \\
\hline $\begin{array}{c}\text { T2-weighted } \\
\text { (long/long) }\end{array}$ & $\begin{array}{c}\text { Short T2 relaxation } \\
\text { (dark) }\end{array}$ & Fibrous Tissue, Cortical Bone \\
\cline { 2 - 3 } & $\begin{array}{c}\text { Long T2 relaxation } \\
\text { (bright) }\end{array}$ & $\begin{array}{c}\text { Neoplastaining substances } \\
\text { (bure Fluid, Inflammation }\end{array}$ \\
\hline
\end{tabular}

is the starting point for acquired knowledge, which was refined for the specific task of tumor segmentation.

\section{Knowledge-Based Systems}

Knowledge is any chunk of information that effectively discriminates one class type from another [20]. In this case, tumor will have certain properties that other brain tissues will not and visa-versa. In the domain of MRI volumes, there are two primary sources of knowledge available. The first is pixel intensity in feature space, which describes tissue characteristics within the MR imaging system (based on a review of literature $[36,15,48])$. The second is image/anatomical space and includes expected shapes and placements of certain tissues within the MR image. As each processing stage is described in Section III., the specific knowledge extracted and its application will be detailed.

\section{System Overview}

A strength of the knowledge-based (KB) systems in [34, 7, 6] has been their "coarseto-fine" operation. Instead of attempting to achieve their task in one step, incremental refinement is applied with easily identifiable tissues located and labeled first, allowing a "focus" to be placed on the remaining pixels. To better illustrate the system's organization, we present it at a conceptual level. Figure 1.2 shows the primary steps in extracting tumor from raw MR data. Section III. describes these steps in more detail.

The system has five primary steps. First a pre-processing stage developed in previous works $[34,7,6,8]$, called Stage Zero here, is used to detect deviations from expected properties within the slice. Slices that are free of abnormalities are not processed further. Otherwise, Stage One extracts the intra-cranial region from the rest of the MR image based on information provided by pre-processing. This creates an image mask of the brain that limits processing in Stage Two to only those pixels contained by the mask.

An initial tumor segmentation is produced in Stage Two through a combination of adaptive histogram thresholds in the $\mathrm{T} 1$ and $\mathrm{PD}$ feature images. Additional nontumor pixels are removed in Stage Three via a "density screening" operation. Density 


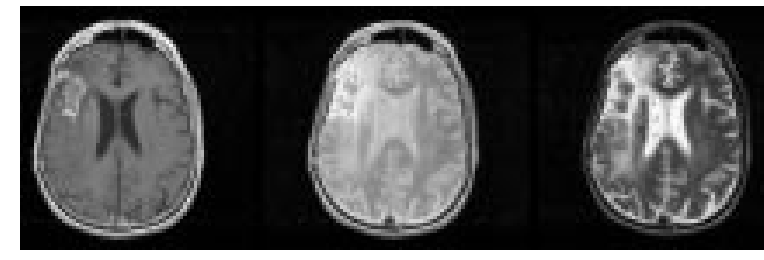

Raw MR image data: T1, PD, and T2-weighted images.

STAGE 0

Pathology Detection.

Slice tissues are located

and tested. Slices with

detected abnormalities

(such as in the white matter class shown) are segmented

for tumor. Slices without

abnormalities are not

processed further.

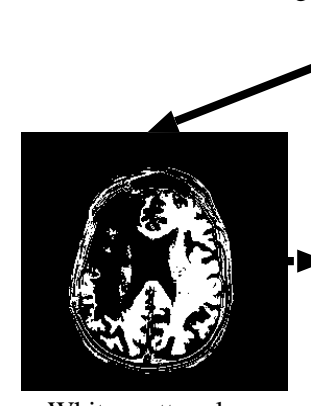

White matter class.

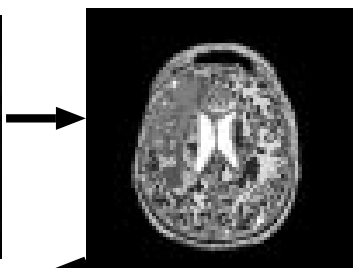

Initial segmentation by unsupervised clustering algorithm.

\section{STAGE ONE}

Intracranial mask created

from initial segmentation

to reclaim possible lost

tumor pixels.

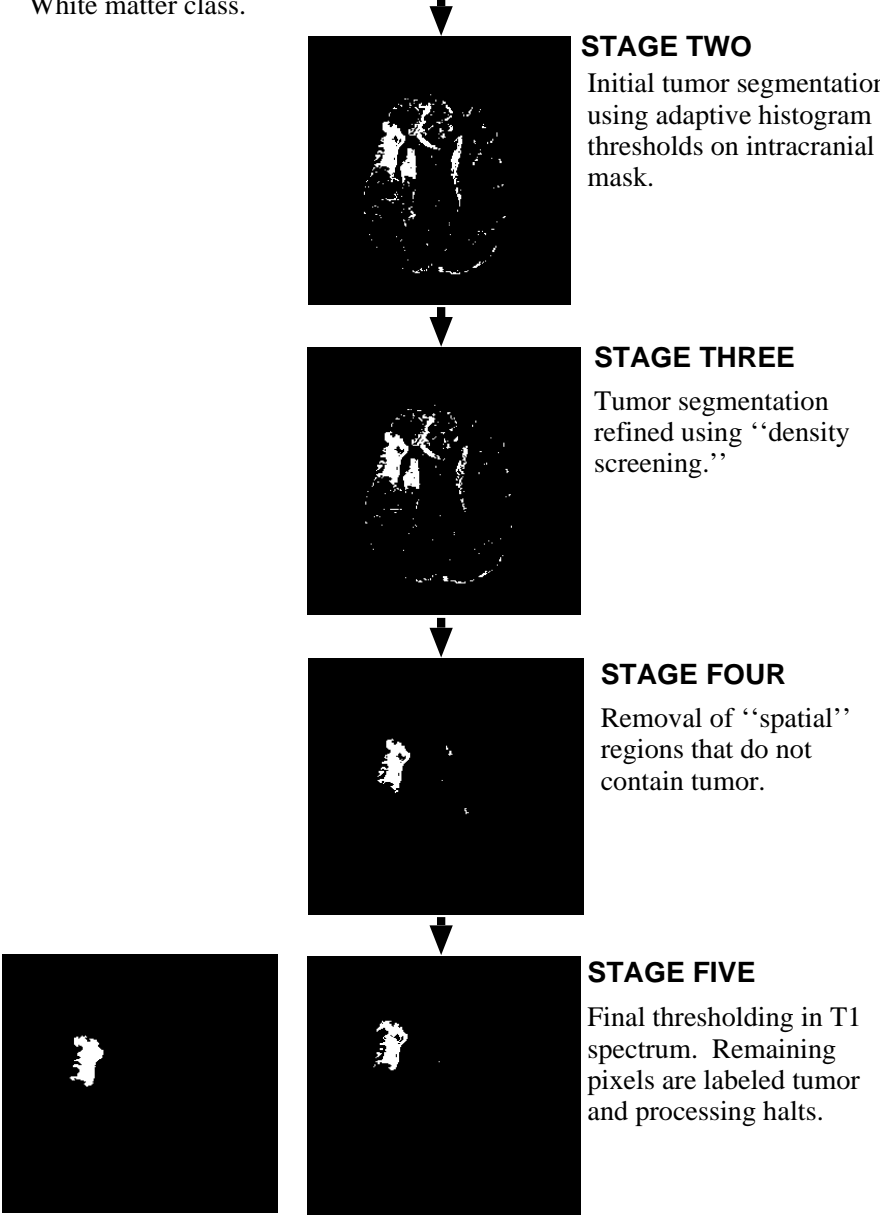

Figure 1.2: System Overview. 


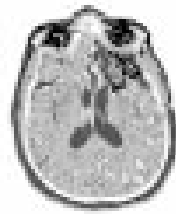

(a)

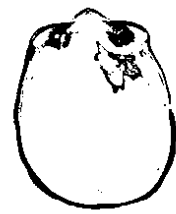

(b)

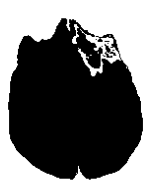

(c)

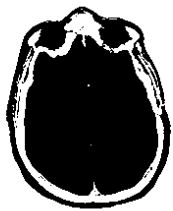

(d)

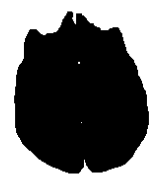

(e)

Figure 1.3: Building the Intra-Cranial Mask. (a) The original FCM-segmented image; (b) pathology captured in Group 1 clusters; (c) intra-cranial mask using only Group 2 clusters; (d) mask after including Group 1 clusters with tumor; (e) mask after extracranial regions are removed.

screening is based on the observation that pixels of normal tissues are grouped more closely together in feature space than tumor pixels.

Stage Four continues tumor segmentation by separately analyzing each spatially disjoint "region" in image space created by a connected components operation. Those regions found to contain no tumor are removed and the remaining regions are passed to Stage Five for application of a final threshold in the T1 spectrum, using the approximated tumor boundary (determined with a fuzzy edge detector). The resulting image is considered the final tumor segmentation and can be compared with a ground truth image.

\section{Classification Stages}

By its nature, tumor is much more difficult to model in comparison to normal brain tissues. Therefore, tumor is defined here more by what it isn't than what it is. Specifically, the tumor segmentation system operates by removing all pixels considered not to be enhancing tumor, with all remaining pixels labeled as tumor.

\section{A. Stage Zero: Pathology Detection}

All slices processed by the tumor segmentation system have been automatically classified as abnormal. They are known to contain glioblastoma-multiforme tumor based on radiologist pathology reports. Since this work is an extension of previous work, knowledge generated during "pre-processing" is available to the tumor segmentation system. Detailed information can be found in $[34,7,6,8]$, but a brief summary is provided.

Slice processing begins by using an unsupervised fuzzy c-means (FCM) clustering algorithm $[5,23]$ to segment the slice. The initial FCM segmentation is passed to an expert system which uses a combination of knowledge concerning cluster distribution in feature space and anatomical information to classify the slice as normal or abnormal. Knowledge implemented in the predecessor system includes rules such as: (1) in a normal slice, CSF belongs to the cluster center with the highest $\mathrm{T} 2$ value in the intra-cranial region; (2) in image space, all normal tissues are roughly symmetrical along the vertical axis (defined by each tissue having approximately the same number of pixels in each brain hemisphere). Abnormal slices are detected by their deviation from "expectations" concerning normal MR slices, such as the one shown in Figure 1.2 whose white matter class failed to completely enclose the ventricle area. An abnormal slice, along with the facts generated in labeling it abnormal, are passed on to the tumor segmentation system. Normal slices have all pixels labeled. 


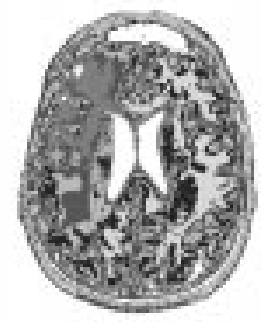

(a)

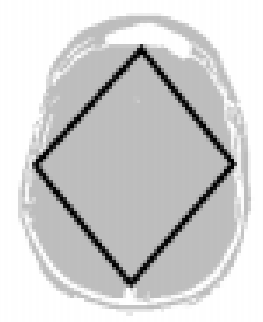

(b)

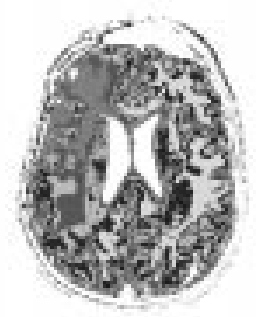

(c)

Figure 1.4: (a) Initial segmented image; (b) a quadrangle overlaid on (a); (c) classes that passed quadrangle test.

\section{B. Stage One: Building the Intra-Cranial Mask}

The first step in the system presented here is to isolate the intra-cranial region from the rest of the image. During pre-processing, extra and intra-cranial pixels were distinguished primarily by separating the clusters from the initial FCM segmentation into two groups: Group 2 for brain tissue clusters, and Group 1 for the remaining extra-cranial clusters. Occasionally, enhancing tumor pixels can be placed into one or more Group 1 clusters with high T1-weighted centroids. In most cases, these pixels can be reclaimed through a series of morphological operations (described below). As shown in Figures 1.3(b) and (c), however, the tumor loss may be too severe to recover morphologically without distorting the intra-cranial mask.

Group 1 clusters with significant "Lost Tumor" can be located, however. During pre-processing, Group 1 and 2 clusters were separated based on the observation that extra-cranial tissues surround the brain and are not found within the brain itself. A "quadrangle" was developed by $\mathrm{Li}$ in $[34,33]$ to roughly approximate the intra-cranial region. Group 1 and 2 clusters were then discriminated by counting the number of pixels a cluster had within the quadrangle. Clusters consisting of extra-cranial tissues will have very few pixels inside this estimated brain, while clusters of intra-cranial tissues will have a significant number. An example is shown in Figure 1.4.

A Group 1 cluster is considered to be have "Lost Tumor" here if more than $1 \%$ of its pixels were contained in the approximated intra-cranial region. The value of $1 \%$ is used to maximize the recovery of lost tumor pixels because extra-cranial clusters with no lost tumor will have very few pixels within the quadrangle, if any at all. Pixels belonging to Lost Tumor clusters (Figure 1.3(b)) are merged with pixels from all Group 2 clusters (Figure 1.3(c)) and set to foreground (a non-zero value), with all other pixels in the image set to background (value $=0$ ). This produces a new intra-cranial mask similar to the one shown in Figure 1.3(d).

Since a Lost Tumor cluster is primarily extra-cranial, its inclusion in the intra-cranial mask introduces areas of extra-cranial tissues, such as the eyes and skin/fat/muscle (Figures 1.3(d)). To remove these unwanted extra-cranial regions (and recover smaller areas of lost tumor, mentioned above), a series of morphological operations [26] are applied, which use window sizes that are the smallest possible (to minimize mask distortion) while still producing the desired result.

Small regions of extra-cranial pixels are removed and separation of the brain from meningial tissues is enhanced by applying a $5 \times 5$ closing operation to the background. Then the brain is extracted by applying an eight-wise connected components operation [26] and keeping only the largest foreground component (the intra-cranial mask). 


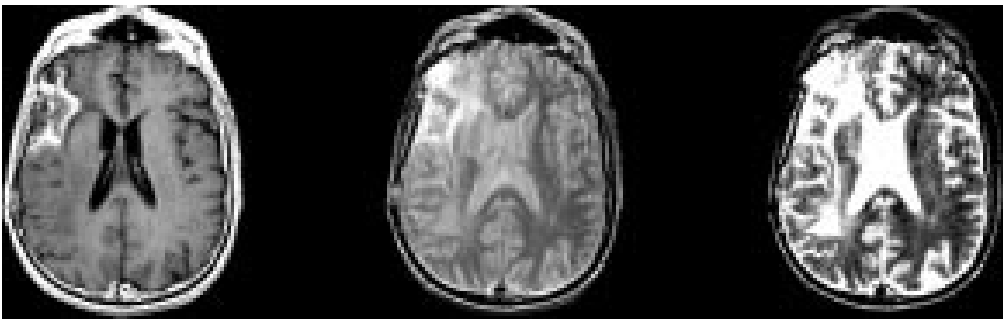

(a)

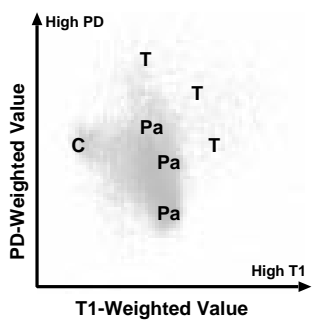

(b)

Figure 1.5: (a) Raw T1, PD, and T2-weighted Data. The distribution of intra-cranial pixels are shown in (b) T1-PD feature space. $\mathrm{C}=\mathrm{CSF}, \mathrm{Pa}=$ Parenchymal Tissues, $\mathrm{T}$ $=$ Tumor

Finally, "gaps" along the periphery of the intra-cranial mask are filled by first applying a $15 \times 15$ closing, then a $3 \times 3$ erosion operation. An example of the final intra-cranial mask can be seen in Figure 1.3(e).

\section{Stage Two: Multi-spectral Histogram Thresholding}

Given an intra-cranial mask, there are three primary tissue types: pathology (which can include gadolinium-enhanced tumor, edema, and necrosis), the brain parenchyma (white and gray matter), and CSF. We would like to remove as many pixels belonging to normal tissues as possible from the mask.

Each MR voxel of interest has a $\langle T 1, P D, T 2\rangle$ location in $\Re^{3}$, forming a featurespace distribution. Based on the fact that pixels belonging to the same tissue type will exhibit similar relaxation behaviors (T1 and T2) and water content (PD), they will then also have approximately the same location in feature space [3]. Figure 1.5(a) shows the signal-intensity images of a typical slice, while (b) shows a histogram for the bivariate features T1/PD with approximate tissue labels overlaid. There is some overlap between classes because the graphs are projections and also due to "partial-averaging" where different tissue types are quantized into the same pixel/voxel.

The typical relationships between enhancing tumor and other brain tissues can also be seen in Figure 1.6, which are histograms for each of the three feature images. These distributions were examined and interviews were conducted with experts concerning the general makeup of tumorous tissue, and the behavior of gadolinium enhancement in the three MRI protocols. From these sources, a set of heuristics were extracted that could be included in the system's knowledge base:

1. Gadolinium-enhanced tumor pixels occupy the higher end of the T1 spectrum.

2. Gadolinium-enhanced tumor pixels occupy the higher end of the PD spectrum, though not with the degree of separation found in T1 space [24].

3. Gadolinium-enhanced tumor pixels were generally found in the "middle" of the $\mathrm{T} 2$ spectrum, making segmentation based on T2 values difficult.

4. Slices with greater enhancement had better separation between tumor and nontumor pixels, while less enhancement resulted in more overlap between tissue types.

Analysis of these heuristics revealed that histogram thresholding could provide a simple, yet effective, mechanism for gross separation of tumor from non-tumor pixels (and thereby an implementation for the heuristics). In fact, in the T1 and PD spectrums, 


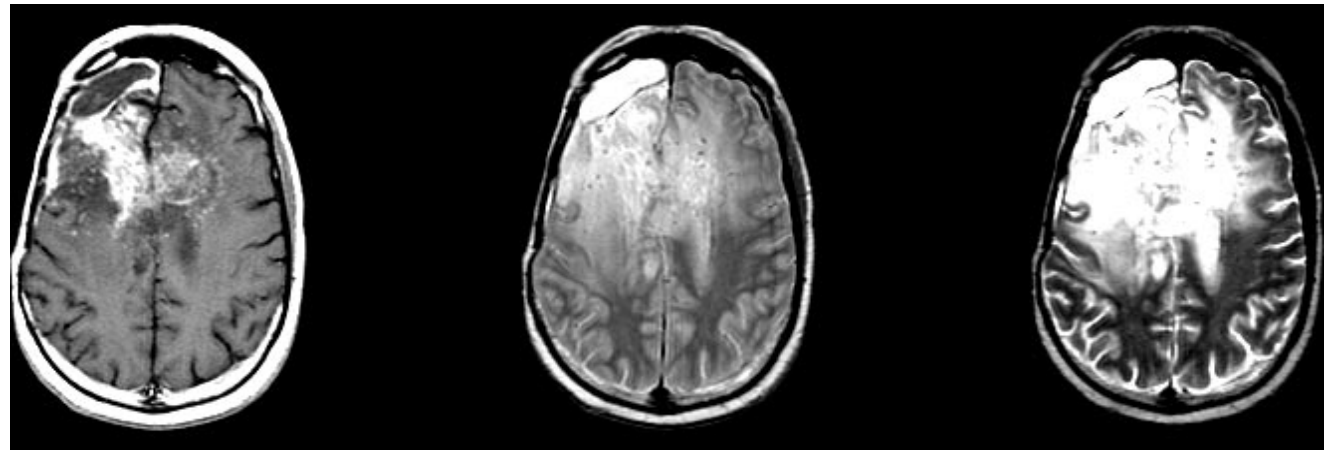

(a) Raw Data

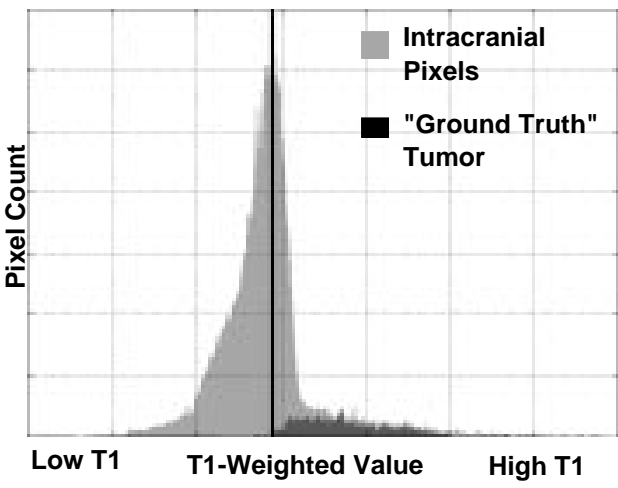

(b) T1-weighted Histogram

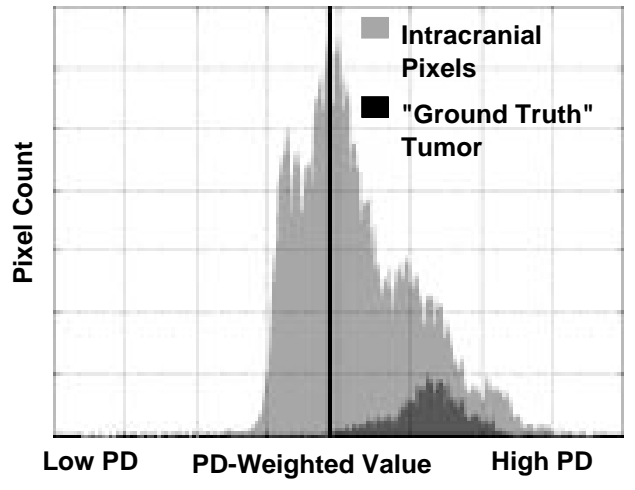

(c) PD-weighted Histogram

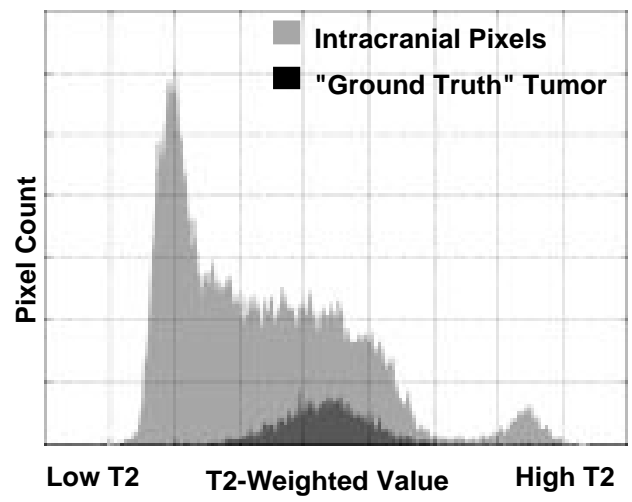

(d) T2-Weighted Histogram

Figure 1.6: Histograms for Tumor and the Intra-Cranial Region. Solid black lines indicates thresholds in T1 and PD-weighted space. 


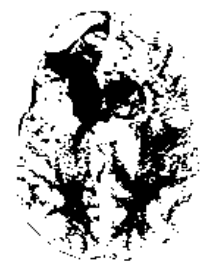

(a)

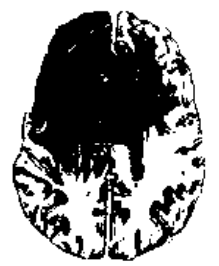

(b)

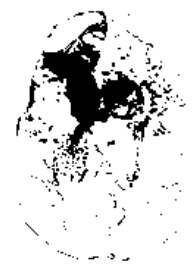

(c)

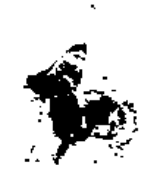

(d)

Figure 1.7: Multi-spectral Histogram Thresholding of Figure 1.6. (a) T1-weighted thresholding; (b) PD-weighted thresholding; (c) Intersection of (a) and (b); (d) Ground truth.

the signal intensity having the greatest number of pixels, that is, the histogram "peaks," were found to be effective thresholds that work across slices, even those with varying degrees of gadolinium enhancement. An example of this is shown in Figure 1.6. The T2 feature had no such property that was consistent across all training slices and was excluded.

For a pixel to survive thresholding, its signal intensity value in a particular feature had to be greater than the intensity threshold for that feature. Figures 1.7(a) and (b) show the results of applying the T1 and PD histogram "peak" thresholds in Figures 1.6(b) and (c). In both of these thresholded images a significant number of nontumor pixels have been removed, but some non-tumor pixels remain in each thresholded image. Since the heuristics listed above state that gadolinium enhanced tumor has a high signal intensity in both the T1 and PD features, additional non-tumor pixels can be removed by intersecting the two images (where a pixel remains only if it's present in both images). An example is shown in Figure 1.7(c).

\section{Stage Three: "Density Screening" in Feature Space}

The thresholding process in Stage Two provides a good initial tumor segmentation, such as the one shown in Figure 1.7(c). Comparing it with the ground truth image Figure 1.7(d), a number of pixels in the initial tumor segmentation are not found in the ground truth image and should be removed. Additional thresholding is difficult to perform, however, without possibly removing tumor as well as non-tumor pixels.

Pixels belonging to the same tissue type will have similar signal intensities in the three feature spectrums. Because normal tissue types have a more or less uniform cellular makeup [36, 15, 48], their distribution in feature space will be relatively concentrated [3]. In contrast, tumor can have significant variance, depending on the local degrees of enhancement and tissue inhomogeneity within the tumor due to the presence of edema, necrosis, and possibly some parenchymal cells captured by the partial-volume effect. Figures 1.5 (b) and (c) show the different spreads in feature space for normal and tumor pixels. Pixels belonging to parenchymal tissues and CSF are grouped more densely by intensity, while pixels belonging to tumor are more widely distributed.

By exploiting this "density" property, non-tumor pixels can be removed without affecting the presence of tumor pixels. Called "density screening," the process begins by creating a 3-dimensional histogram for all pixels remaining in the initial tumor segmentation image after thresholding. The histogram array itself has a T1_range $\times$ $P D \_r a n g e \times T 2 \_r a n g e$ size of $128 \times 128 \times 128$ intensity bins. For each feature, the maximum and minimum signal intensity values in the initial tumor segmentation are found 


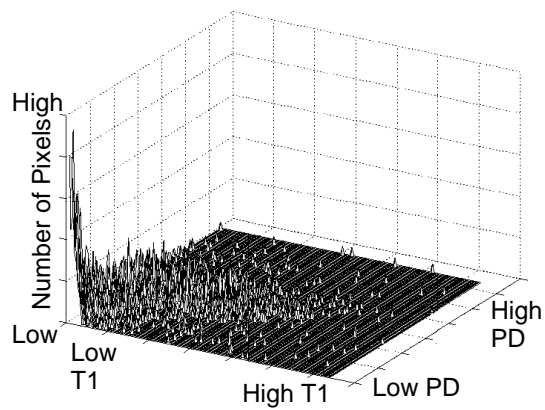

(a) 2D-Histogram Projection

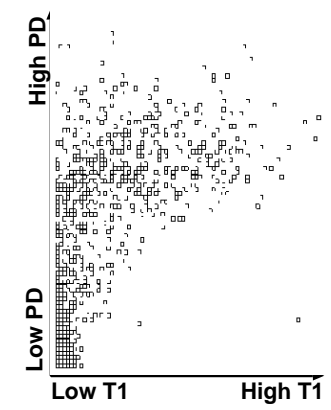

(b) Scatterplot

Before Screening

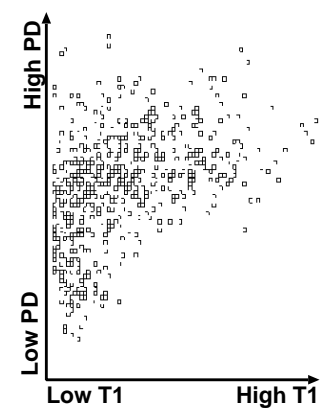

(c) Scatterplot

After Screening

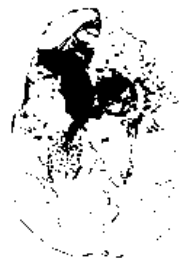

(d) Initial Tumor

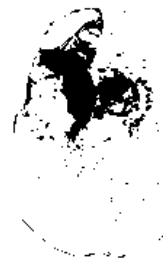

(e) Removed Pixels (Black) (f) Ground Truth

Figure 1.8: Density Screening Initial Tumor Segmentation From Figure 1.7(c).

and quantized into the histogram array (i.e., the minimum $\mathrm{T} 1$ intensity value occupies $\mathrm{T} 1$ Bin 1, the maximum T1 intensity value occupies T1 Bin 128), with all T1 values in between "quantized" into one of the 128 bins. This quantization was done for two reasons. First, sizes of a three-dimensional histogram quickly became prohibitively large to store and manipulate. Even a $256^{3}$ histogram has nearly 17 million elements. Secondly, levels of quantization can make the "dense" nature of normal pixels more apparent while still leaving tumor pixels relatively spread out. For the 12-bit data studied here, after thresholding, slices had a range of approximately 800 intensity values in each feature. The actual value of 128 was empirically selected. Using 64 bins blurred the separation of tumor and non-tumor pixels in training slices where the tumor boundary was not as well defined. Values similar to 128 , such as 120 or 140 , are unlikely to significantly change the "quantization" effect and should yield similar results. The histograms and scatterplots shown in Figure 1.8 were created using 128 bins.

From the 3D histogram, three 2D projections are generated: T1/PD, T1/T2, and $\mathrm{PD} / \mathrm{T} 2$. An example 2D projection is shown in Figure 1.8(a), which was generated from the slice shown in Figure 1.7(c). A corresponding scatterplot is shown in Figure 1.8(b). The bins with the most pixels (the highest "peaks" in Figure 1.8(a)) can be seen in the lowest T1/PD corner and are comprised of non-tumorous pixels that should be removed. In contrast, tumor pixels, while greater in number, are more widespread and have lower peaks in their bins.

In each projection, the highest peak is found and designated as the starting point for a region growing [27] process that will "clear" any neighboring bin whose cardinality (number of pixels in that bin) is greater than a set threshold $(\mathrm{T} 1 / \mathrm{PD}=3, \mathrm{~T} 1 / \mathrm{T} 2=4$, $\mathrm{PD} / \mathrm{T} 2=3$ ). This will result in a new scatterplot similar to that shown in Figure 1.8(c). A pixel is removed from the tumor segmentation if it is located in a bin that has been 


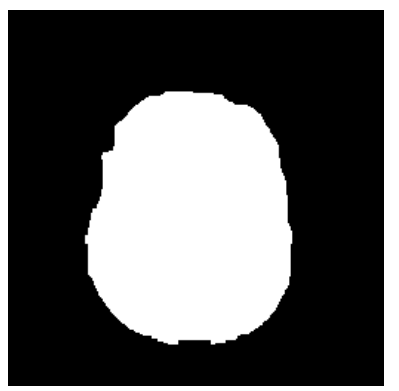

(a)

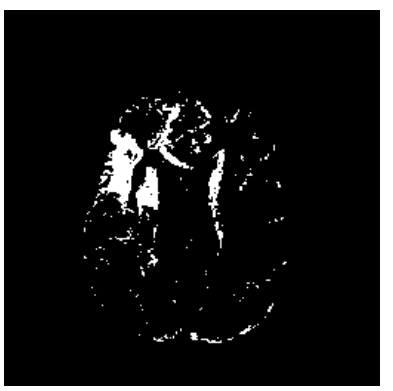

(b)

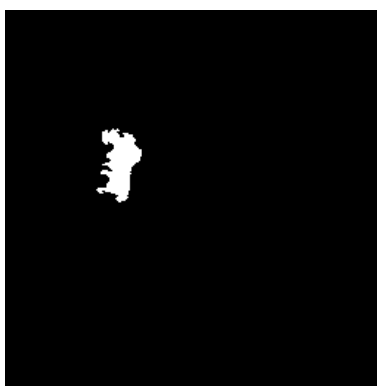

(c)

Figure 1.9: Regions in Image Space. After processing the intra-cranial mask (a), (b) is an initial tumor segmentation. Only one region, as shown in the ground-truth image (c) is actual tumor. Region analysis discriminates between tumorous and non-tumorous regions.

"cleared" in any of the three feature-domain projections. Figures 1.8(d) and (e) are the tumor segmentation before and after the entire density screening process is completed. Note that the resulting image is closer to ground truth.

The thresholds used were determined from training slices by creating a $3 \mathrm{D}$ histogram, including $2 \mathrm{D}$ projections, using only pixels contained in the initial tumor segmentation. Then the ground truth tumor pixels for each slice were overlaid on the respective projections. So, given a 3D histogram of an initial tumor segmentation, all pixels not in the ground truth image are removed, leaving only tumor behind without changing the dimensions and quantization levels of the histogram. The respective 2D projections of all training slices were examined. It was found that the smallest bin cardinality bordering a bin occupied by known non-tumor pixels made an accurate threshold for the given projection.

\section{E. Stage Four: Region Analysis and Labeling}

In Stages Two and Three, the knowledge extracted up to this point was applied to pixels individually. Stage Four, allows spatial information to be introduced by considering pixels on a region or component level. Applying an eight-wise connected components operation [26] to the refined tumor segmentation generated by Stage Three, allows each region to be tested separately for the presence of tumor. An example is shown in Figure 1.9. After processing the intra-cranial mask shown in Figure 1.9(a) in Stages Two and Three, a refined tumor segmentation (b) is produced. The segmentation shows a number of spatially disjoint areas, but ground truth tumor in Figure 1.9(c) shows that only one region actually contains tumor. Therefore, decisions must be made regarding which regions contain tumor and which do not.

\section{Removing Meningial Regions}

In addition to tumor, meningial tissues immediately surrounding the brain, such as the dura or pia mater, receive gadolinium infused blood. As a result they can have a high T1 signal intensity that may violate with the knowledge base's assumption in Section 2. that regions with the highest T1 value are most likely tumor. These extracranial tissues can be identified and removed via anatomical knowledge by noting that since they are thin membranes, meningial regions should lie along the periphery of the brain in a relatively narrow margin.

Figure 1.10 shows that an approximation of the brain periphery can be used to detect meningial tissues. The unusual shape of the intra-cranial region is due to prior 


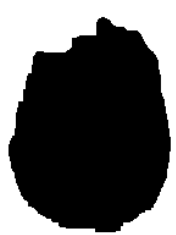

(a)

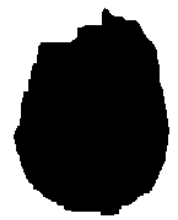

(b)

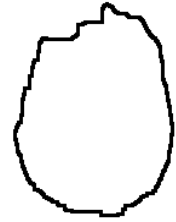

(c)

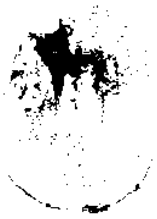

(d)

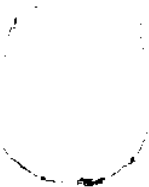

(e)

Figure 1.10: Removing Meningial Pixels. A "ring" that approximates the brain periphery is created by applying a $7 \times 7$ erosion operation to the intra-cranial mask (a), resulting in image (b). Subtracting (b) from (a), creates a "ring", shown in (c). By overlaying this "ring" onto a tumor segmentation (d), small regions of meningial tissues (e) can be detected and removed. The unusual shape of the intra-cranial region is due to prior resection surgery.

resection surgery. The periphery is created by applying a $7 \times 7$ erosion operation to the intra-cranial mask and subtracting the resultant image from the original mask, as shown in Figure 1.10(a-c). Each component or separate region in the refined tumor mask is now intersected with the brain periphery. Any region which has more than $50 \%$ of its pixels contained in the periphery is marked as meningial tissue and removed. Figure 1.10(d) shows a tumor segmentation which is intersected with the periphery from Figure 1.10(c). In Figure 1.10(e), the pixels that will be removed by this operation are shown and they are indeed meningial pixels.

\section{Removing Non-Tumor Regions}

Once any extra-cranial regions have been removed, the knowledge base is applied to discriminate between regions with and without tumor based on statistical information about the region. A region mean, standard deviation, and skewness in $\langle T 1\rangle,\langle P D\rangle$, and $\langle T 2\rangle$ feature space respectively are used as features. The concept exploited is that trends and characteristics described at a pixel level in Section C. are also applicable on a region level. By sorting regions in feature space based upon their mean values, rules based on their relative order can be created:

1. Large regions that contain tumor will likely contain a significant number of pixels that are of highest intensity in T1 and PD space, while regions without tumor likely contain a significant number of pixels of lowest intensity in T1 and PD space.

2. The means of regions with similar tissue types neighbor one another in feature space.

3. The intra-cranial region with the highest mean $\mathrm{T} 1$ value and a "high" $\mathrm{PD}$ and $\mathrm{T} 2$ value, is considered "First Tumor," against which all other regions are compared.

4. Other regions that contain tumor are likely to fall within 1 to 1.5 standard deviations (depending on region size) of First Tumor in T1 and PD space.

While most glioblastoma-multiforme tumor cases have only one tumorous spatially compact region that has the highest mean $\mathrm{T} 1$ value, in some cases, the tumor has grown such that it has branched into both hemispheres of the brain, causing the tumor to appear disjoint in some slices, or it has fragmented as a result of treatment. Also, 


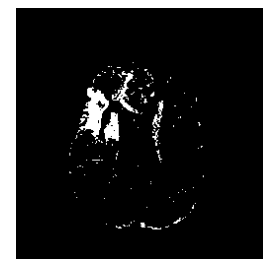

(a)

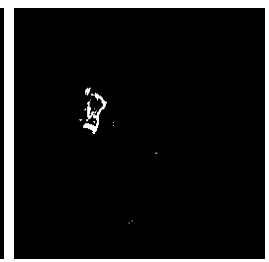

(b)

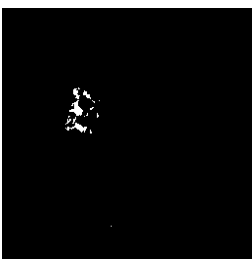

(c)

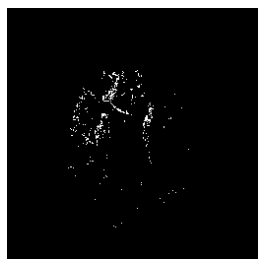

(d)

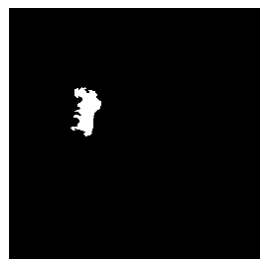

(e)

Figure 1.11: Using Pixel Counts to Remove Non-Tumorous Regions. Given a refined tumor segmentation after Stage Three (a), spatial regions with a significant number of pixels highest in T1 space (b) or PD space (c) are likely to contain tumor. Regions with pixels lowest in T1 space (d) are unlikely to contain significant tumor. Ground truth is shown in (e).

Table 1..2: Region Labeling Rules Based on Pixel Presence.

\begin{tabular}{||l|l|c||}
\hline Region Size & Pixels in intersections with the 3 masks & Action \\
\hline \hline$\leq 5$ & $\begin{array}{l}\text { Any Bottom T1 Pixels AND } \\
\text { Less than 2 Top T1 Pixels }\end{array}$ & $\begin{array}{c}\text { Remove } \\
\text { Non-Tumor }\end{array}$ \\
\hline$\geq 500$ & More than RegionSize $\times 0.06$ Top T1 Pixels & $\begin{array}{c}\text { Label As } \\
\text { Tumor }\end{array}$ \\
\hline$\geq 5$ & $\begin{array}{l}\text { No Top T1 Pixels AND } \\
\text { More Than RegionSize } \times 0.005 \text { Bottom T1 Pixels AND } \\
\text { Less Than RegionSize } \times 0.01 \text { Top PD Pixels }\end{array}$ & \\
\hline
\end{tabular}

different tumor regions do not enhance equally. Thus, cases can range from a single well-enhancing tumor to a fragmented tumor with different levels of enhancement. In comparison, the makeup of non-tumor regions is generally more consistent than in tumorous regions. Therefore, the knowledge base is designed to facilitate removal of nontumor regions because their composition can be more reliably modeled and detected.

Regions that comply with the first heuristic listed above are the easiest to locate and their statistics can be used to examine the remaining regions. To apply the first heuristic, three new image masks are created. The first image mask takes the refined tumor segmentation image and keeps only $20 \%$ of the highest T1 value pixels (i.e., if there were 100 pixels in the refined tumor image, the 20 pixels with the highest T1 values are kept). The second mask keeps the highest $20 \%$ in PD space, while the third mask keeps the $30 \%$ lowest in T1 space. Each region is isolated and intersected with each of the 3 masks created. The number of pixels of the region in a particular mask is recorded and compared with the rules listed in Table 1..2. An example is shown in Figure 1.11.

Regions that do not activate any of the rules in Table $1 . .2$ remain unlabeled and are analyzed using the last two heuristics.

According to the third heuristic, given a region that has been positively labeled tumor as a point of reference, a search can be made in feature space for neighboring tumor regions. Normally, the region with the highest T1 mean value can be selected as this point of reference (called "First Tumor"). To guard against the possibility that an extra-cranial region (usually meningial tissues at the inter-hemispheric fissure) has been selected instead, the selected region is verified via the heuristic that a tumor region will not only have a very high T1 mean value, but will also occupy the highest half of all 
Table 1..3: Region Labeling Rules Based on Statistical Measurements. Largest is the largest known tumor region.

(a) Rules Based on Standard Deviation (SD) of "First Tumor"

\begin{tabular}{|c|c|c|}
\hline Region Size & If Region's Mean Values are: & Action \\
\hline $\begin{array}{l}\leq 10 \text { OR } \\
\geq \text { Largest } / 4\end{array}$ & $\begin{array}{l}\text { More than } 1 \text { SD away in T1 space OR } \\
\text { More than } 1 \text { SD away in PD space. }\end{array}$ & Remove \\
\hline $\begin{array}{l}\geq 10 \mathrm{AND} \\
\leq \text { Largest } / 4\end{array}$ & $\begin{array}{l}\text { More than } 1.5 \mathrm{SD} \text { away in T1 space AND } \\
\text { More than } 1.5 \mathrm{SD} \text { away in PD space. }\end{array}$ & Remove \\
\hline \multicolumn{3}{|c|}{ (b) Labeling Rules Based on Region Statistics } \\
\hline$\geq 100$ & $\begin{array}{l}\text { Region } T 1 \text { Skewness } \leq 0.75 \text { AND } \\
\text { Region } P D \_ \text {Skewness } \leq 0.75 \text { AND } \\
\text { Region } T 2 \_ \text {Skewness } \leq 0.75\end{array}$ & Remove \\
\hline
\end{tabular}

regions in sorted $\mathrm{PD}$ and $\mathrm{T} 2$ mean space. For example, if there were 10 regions total, the region being tested must be one of the 5 highest mean values in both $\mathrm{PD}$ and T2 space. If the candidate region passes, it is confirmed as First Tumor. Otherwise, it is discarded and the region with the next highest T1 mean value is selected for testing as First Tumor.

Once First Tumor has been confirmed, the search for neighboring tumor regions can begin. Although tumorous regions can have between-slice variance, the third and fourth heuristics hold for the purpose of separating tumor from non-tumor regions within a given slice. Furthermore, the standard deviations in T1 and PD space of a known tumor region were found to be a useful and flexible distance measure.

Table 1..3(a) lists the two rules that used the standard deviation to remove nontumor regions, based on the size (number of pixels) of the region being tested. The rule in Table 1...3(b) serves as a tie-breaker for some regions that were not labeled before. The term Largest is used to indicate the largest known tumor region. In most cases there was only a single tumor region, so the "first tumor" region was also the Largest region. In cases where tumor was fragmented, however, a larger tumorous region would have a greater number of pixels (and thus, more reliable sample) to calculate the mean and standard deviation for the distance measure. Therefore, the system would find Largest by searching for the largest region that was within one standard deviation in both T1 and PD space to the First Tumor region. After the rules in Table 1..3 are applied, all regions that were not removed are kept as regions containing tumor.

\section{F. Stage Five: Final T1 Threshold}

At the end of Stage Four, the regions with no tumor have been removed, but nontumor pixels may still be found in those regions considered to contain tumor. While enhancing tumor has properties in each of the three available features that have been used as knowledge, discussions with an expert radiologist [39] have indicated that final tumor boundaries are determined by pixel intensities in the T1-weighted image. Thresholds were described in Section C. in a relatively coarse manner because the boundary of enhancing tumor was "obscured" by pixels belonging to non-tumor tissues. With the removal of most of these non-tumor tissues in Stages Two through Four, however, a greater level of focus can be placed and a more precise threshold can be applied.

The threshold is determined using the principle that the spatial boundary between enhancing tumor and surrounding tissues contain pixels whose signal intensities correspond to the tumor/non-tumor boundary in T1-weighted space. One of the most common methods of isolating spatial boundaries between objects of interest (with dif- 
ferent intensities) has been edge detection. In our case, edges represent differences in T1-weighted signal intensities, the more distinct boundary a tumor has, the greater its edge strength will be.

Most edge-detection based methods, such as those in [21, 12, 45, 22, 60, 59, 2] attempt to use edges to trace the tumor's contours. This can work well for tumors with distinct boundaries, as shown in Figure 1.12(d) using a standard Sobel operator, but can have significant problems with more diffuse tumors, shown in Figure 1.12(i). A variety of edge detection operators have been introduced, such as Canny and Bergholm [27]. Most of these, however, have a number of parameters that are difficult to automatically optimize, especially in a domain where the object of interest can have such wide ranging characteristics [14]. As a result, Dellepiane [12] and Raff and Newman [45] have suggested that edge detection is unlikely to work reliably for complex structures like tumors.

Edge detection may still provide knowledge to be exploited, however. By noting that edges not only approximate the tumor boundary spatially, but can also indicate the approximate signal intensity of that boundary which can be used in a threshold operation. Now detected edges need not be perfect, merely sufficient to indicate the appropriate signal intensity. Edge detection must still be reasonable, however, for the method to work.

To address the problem of detecting edges in tumors with diffuse, and to minimize the problem of parameter optimization, the technique introduced here uses a "fuzzy" approach to edge detection presented by Tao and Thompson in [49] which used fuzzy if-then rules that were based on the relationship between each pixel and its eight-wise neighbors. Structure elements, sixteen in total with examples shown in Figure 1.13, are used to develop a fuzzy if-then rule:

IF [the differences ( $D_{x}$ 's) between the intensities of the pixels (marked with "x") and the center pixel are small] AND [the differences ( $D$ 's) between the intensities of the pixels (not marked with " $\mathrm{x}$ ") and the center pixel are large] THEN the center pixel of this structure is an edge pixel.

The authors state that the fuzzy memberships small and large are defined with a bell-shaped function, though they do not specify a particular function. Here, a Gaussianbased function is used and the fuzzy set small is defined as:

$$
\mu_{\text {small }}=e^{-\frac{\operatorname{Diff}_{(a, b)}^{2}}{2 \sigma^{2}}}
$$

where $\operatorname{Diff} f_{(a, b)}$ is the absolute intensity difference between the center pixel and the eight-wise neighbor $(\mathrm{a}, \mathrm{b})$. The fuzzy set large is defined as: $\mu_{\text {large }}=1-\mu_{\text {small }}$. The actual Gaussian formula is not used to allow a membership of $\mu_{\text {small }}=1.0$ to be returned when Diff $=0$. Also, in a standard Gaussian function, the value $\sigma$ represents the standard deviation. For defining the fuzzy set, it controls how quickly $\mu_{\text {small }}$ decreases (and $\mu_{\text {large }}$ increases) as the intensity difference, Diff, becomes larger. In this preliminary study, $\sigma=2.0$, though it could be possible to have rules in the knowledge-base adjust the value according to a tumor's characteristics.

The fuzzy if-then rule described above is used to determine a pixel's "edge potential" (PEP) within a given edge structure (Figure 1.13) by calculating the memberships between the center pixel and each of it eight-wise neighbors for the fuzzy set small (if the neighbor is covered by an " $\mathrm{x}$ " in the edge structure) or large (if the neighbor is uncovered), and returning the minimum membership. For example, using the first structure (PEP1) in Figure 1.13, its edge potential would be:

$\mu_{P E P 1}(x, y)=\min \left(\mu_{\text {small }}\left(\operatorname{Diff}_{(x-1, y-1)}\right), \mu_{\text {small }}\left(\operatorname{Diff}_{(x-1, y-1)}\right), \mu_{\text {small }}\left(\operatorname{Diff} f_{(x-1, y)}\right)\right.$,

$\mu_{\text {small }}\left(\operatorname{Diff}_{(x-1, y+1)}\right), \mu_{\text {small }}\left(\operatorname{Diff} f_{(x, y-1)}\right), \mu_{\text {small }}\left(\operatorname{Diff} f_{(x, y+1)}\right)$, 


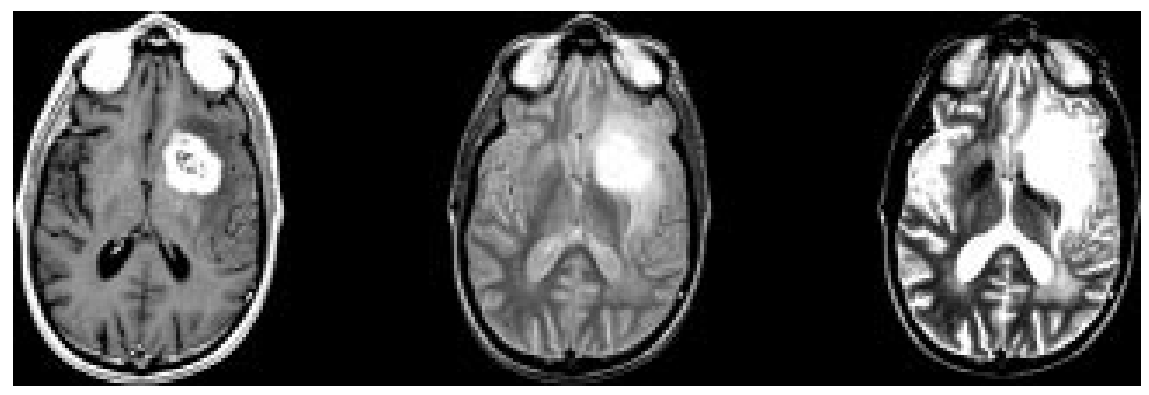

(a) Raw Data

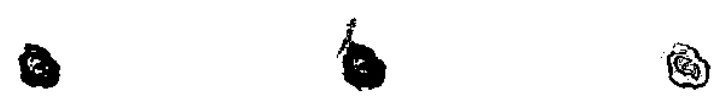

(6)
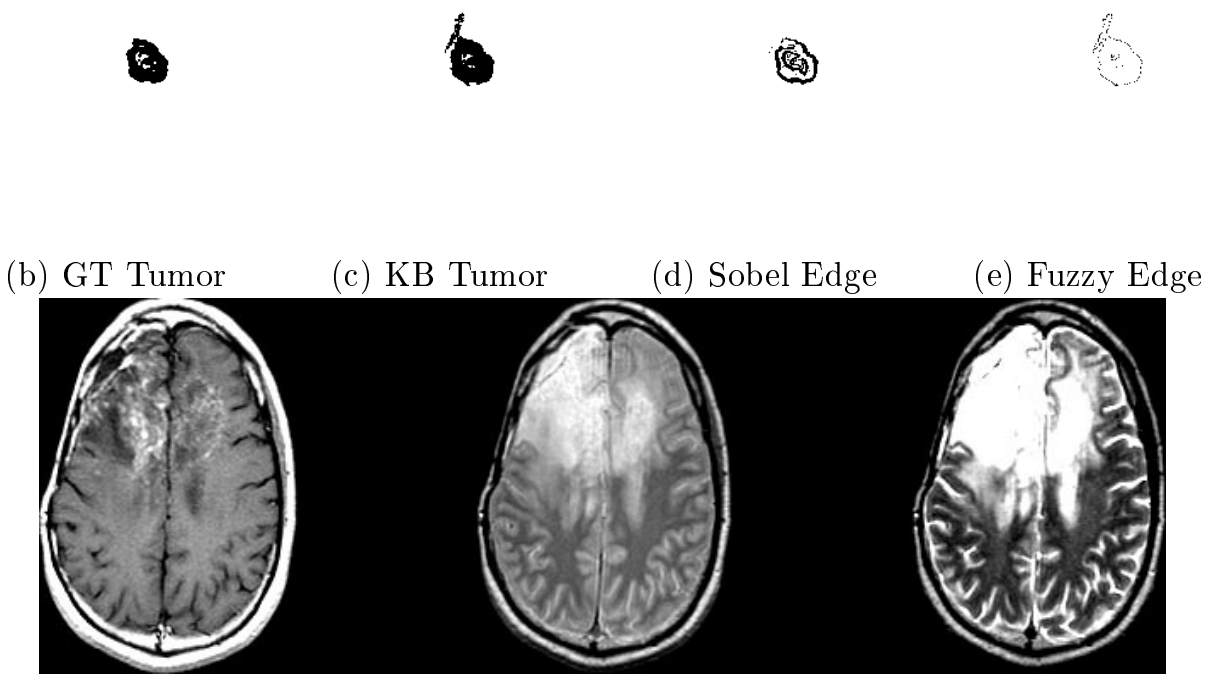

(f) Raw Data
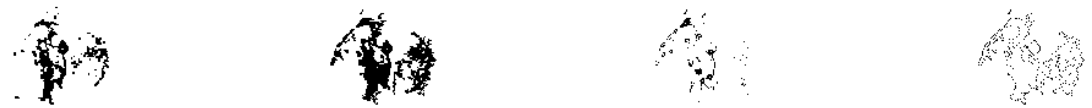

(g) GT Tumor

(h) KB Tumor

(i) Sobel Edges

(j) Fuzzy Edges

Figure 1.12: Detecting Edges Along Tumor Boundaries. Given a tumor segmentation mask, (b) and (h), produced by Stage Four, edge detection is performed on pixels contained by the mask to find the tumor's boundaries. The results of a Sobel operator are shown in (d) and (i), while (e) and (j) show the results of a fuzzy edge detector described in [49]. The tumor in (a) has distinct edges and both edge detectors work well, though the Sobel operator more closely matches ground truth (b). The tumor in (e), however, performs relatively poorly, for both the Sobel and fuzzy edge detectors. 

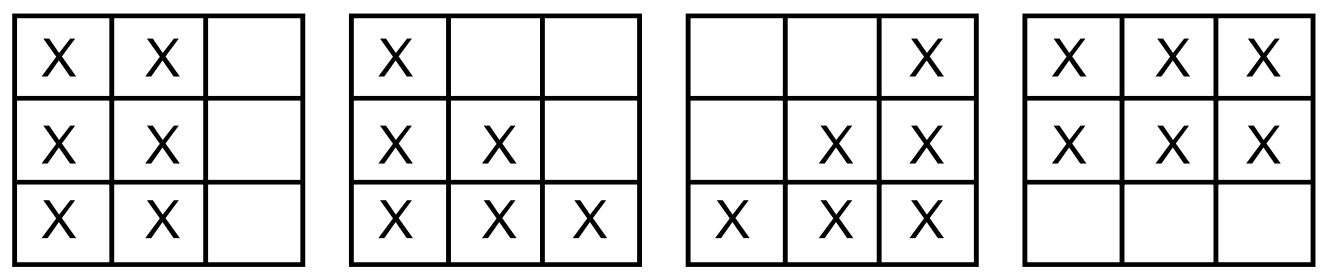

Figure 1.13: Edge Structures for Fuzzy Edge Detection. Edge structures, examples shown above, are used to generate the fuzzy if-then rules. Neighboring pixels covered by an " $\mathrm{x}$ " are used to calculate membership in the fuzzy set small, while those uncovered are used to calculate membership in the fuzzy set large.

$$
\left.\mu_{\text {large }}\left(\operatorname{Diff} f_{(x+1, y-1)}\right), \mu_{\text {large }}\left(\operatorname{Dif} f_{(x+1, y)}\right), \mu_{\text {large }}\left(\operatorname{Diff} f_{(x+1, y+1)}\right)\right)
$$

Given sixteen edge structures, a pixel will have sixteen possible edge memberships. The pixel's final edge membership is set by keeping the membership of the structure the best matched the edge (i.e., the structure with the highest membership). Formally:

$$
P E P(x, y)=\max \left(\mu_{P E P 1}(x, y), \ldots, \mu_{P E P 16}(x, y)\right)
$$

Once final edge memberships have been calculated for all pixels, the detected edges are "thinned" by removing redundant edge pixels through a local maxima operation. A "pseudo-centroid" of the remaining edge strengths is then calculated and only those edges that are stronger than the pseudo-centroid are kept, producing a final edge image, similar to those shown in Figures 1.12(f) and (j).

The method proposed by Tao and Thompson was implemented as described in [49] with the addition that the technique only considers pixels contained in an image mask (in this case, the tumor segmentation mask produced at the end of Stage Four). Once the final edge image is produced, the final $\mathrm{T} 1$ threshold is calculated by averaging the $\mathrm{T} 1$ signal intensities of all pixels contained in the final edge image. During averaging, the signal intensities are not weighted by edge strength as it did not significantly affect the final T1 threshold. Once the T1 threshold is calculated, it is applied to the tumor segmentation image produced at the end of Stage Four (keeping only those pixels whose $\mathrm{T} 1$ signal intensity is greater than the $\mathrm{T} 1$ threshold). The resultant image is considered the final tumor segmentation and processing halts.

\section{Results}

A total of 385 slices from 33 volumes across 8 patients diagnosed with glioblastomamultiforme tumor, who also underwent radiation and chemo-therapy treatment, were available for processing. From this data set, 65 training slices, shown in Table 1..4, were extracted and used to construct the knowledge base and rules.

After processing by the system, the knowledge-based tumor segmentations were compared with radiologist-verified "ground-truth" tumor segmentations [56], as well as three supervised methods. To measure how well (on a pixel level) a tumor segmentation compares with ground truth, two metrics were used. The first, "Percent Match," is simply the number of true positives divided by the total tumor size. The second, is called a "Correspondence Ratio," and was created to account for the presence of false positives:

$$
\text { Correspondence Ratio }=\frac{\text { True Pos. }-(0.5 * \text { False Pos. })}{\text { Number Pixels in Ground Truth Tumor }}
$$


Table 1..4: MR Slice Distribution. Parenthesis indicate the number of slices from that volume that were used as training. $\mathrm{Rn}=$ Repeat Scan $\mathrm{n}$.

\begin{tabular}{|c|c|c|c|c|c|c|c|c|}
\hline & \multicolumn{7}{|c|}{ \# Slices Extracted from Patient Volume } \\
\hline Scan & P1 & P2 & P3 & P4 & P5 & P6 & P7 & P8 \\
\hline \hline Base & 10 & $13(13)$ & 12 & $16(1)$ & $9(9)$ & 15 & $12(7)$ & 7 \\
\hline R1 & $11(1)$ & $14(14)$ & 12 & $15(1)$ & 10 & 12 & & \\
\hline R2 & 11 & $15(2)$ & - & $15(15)$ & $8(1)$ & 13 & - & - \\
\hline R3 & 10 & - & - & - & $8(1)$ & 15 & - & - \\
\hline R4 & 12 & - & - & - & $7(1)$ & 15 & - & - \\
\hline R5 & - & - & - & - & - & 16 & - & - \\
\hline R6 & - & - & - & - & - & 15 & - & - \\
\hline R7 & - & - & - & - & - & 14 & - & - \\
\hline R8 & - & - & - & - & - & 14 & - & - \\
\hline R9 & - & - & - & - & - & 11 & - & - \\
\hline R10 & - & - & - & - & - & 14 & - & - \\
\hline R11 & - & - & - & - & - & 18 & - & - \\
\hline R12 & - & - & - & - & - & 18 & - & - \\
\hline \hline Total & $54(1)$ & $42(29)$ & 24 & $46(16)$ & $42(12)$ & 187 & $12(7)$ & 7 \\
\hline
\end{tabular}

For comparing on a per volume basis, the average value for Percent Match was generated using:

$$
\text { Average } \% \text { Match }=\frac{\sum_{i=1}^{\text {slices in set }}(\% \text { match })_{i} \times(\text { number ground truth pixels })_{i}}{\sum_{i=1}^{\text {slices in set }}(\text { number ground truth pixels })_{i}}
$$

The average value for the Correspondence Ratio is similarly generated.

\section{A. Knowledge-Based vs. Supervised Methods}

Table 1..5 shows how well the knowledge-based system performs against the $\mathrm{k}$ nearest neighbors $(\mathrm{kNN})$ algorithm $(\mathrm{k}=7)$ [11] method for both the Percent Match and Correspondence Ratio metrics. The $\mathrm{kNN}$ numbers shown were averaged over multiple trials of ROI selection, meaning that all $\mathrm{kNN}$ slice segmentations were effectively training slices. Multiple trials/observers also introduces the question of inter and intra-observer variability. This is noted in Table $1 . .5$ by calculating "SDP," the standard deviation as a percentage of tumor volume (for each volume). In some volumes, variability was as high as $40 \%$ of tumor volume. In contrast, the knowledge-based system was built from a small subset of the available slices and processed over 300 slices in unsupervised mode with a static rule set, allowing for complete repeatability. Table 1..5, shows that the knowledge-based system has a higher Percentage Match value (indicating it captured more of the ground truth tumor) in 30 of 33 volumes, and outperformed kNN in Correspondence Ratio in 31 of 33 volumes.

It must be noted, however, that many of the false positives affecting kNN's Correspondence Ratio values are extra-cranial pixels. The kNN method was applied to the whole image and no extraction of the actual tumor is done, which would require additional supervisor intervention, especially in patients with tumor that has "fragmented" as a result of treatment. Some of these kNN volumes had the tumor manually extracted in $[52,58,53]$, using the "best" segmentation from the multiple trials, and are shown in Table A.. Only total tumor volumes were available, so Percent Match and Correspondence Ratios could not be calculated for these volumes. In all 10 volumes listed in 
Table 1..5: Tumor Volume Comparison (Pat. = Patient; Vol. $=$ Scan Volume; $\mathrm{KB}=$ Knowledge Based; $\% \mathrm{M}=$ Percent Match; $\mathrm{CR}=$ Correspondence Ratio; $\mathrm{SDP}=$ Standard Deviation as a Percentage of Total Tumor Volume; \# Tr. = Number of Trials; \# Obs. $=$ Number of Observers)

\begin{tabular}{|c|c|c|c|c|c|c|c|c|c|}
\hline \multirow[b]{2}{*}{ Pat. } & \multirow[b]{2}{*}{ Vol. } & \multirow{2}{*}{$\begin{array}{c}\text { GT } \\
\text { Volume }\end{array}$} & \multicolumn{2}{|c|}{ KB } & \multicolumn{5}{|c|}{ kNN } \\
\hline & & & $\% \mathrm{M}$ & $\mathrm{CR}$ & $\% \mathrm{M}$ & $\mathrm{CR}$ & SDP & \# Tr. & \# Obs \\
\hline \multirow[t]{5}{*}{1} & Base & 7213 & 0.98 & 0.87 & 0.89 & 0.64 & 0.07 & 5 & 2 \\
\hline & R1 & 7240 & 0.98 & 0.86 & 0.89 & 0.52 & 0.19 & 5 & 2 \\
\hline & R2 & 7470 & 0.98 & 0.80 & 0.88 & 0.54 & 0.14 & 5 & 2 \\
\hline & R3 & 6395 & 0.98 & 0.84 & 0.88 & 0.47 & 0.40 & 5 & 2 \\
\hline & R4 & 6560 & 0.97 & 0.79 & 0.83 & 0.32 & 0.07 & 5 & 2 \\
\hline \multirow[t]{3}{*}{2} & $\overline{\overline{\text { Base }}}$ & $\bar{~} 12230$ & $\overline{0.91}$ & 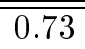 & 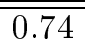 & $\overline{0} 0.51$ & 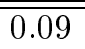 & $\overline{5}$ & $\overline{22}$ \\
\hline & R1 & 14609 & 0.76 & 0.70 & 0.52 & 0.29 & 0.16 & 5 & 2 \\
\hline & $\mathrm{R} 2$ & 20924 & 0.80 & 0.73 & 0.49 & 0.25 & 0.13 & 5 & 2 \\
\hline \multirow[t]{2}{*}{3} & 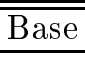 & $\begin{array}{l}10892 \\
\end{array}$ & $\begin{array}{c}0.92 \\
\end{array}$ & 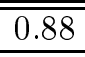 & 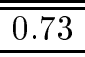 & $\begin{array}{c}0.46 \\
\end{array}$ & 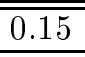 & $\overline{5}$ & 3 \\
\hline & $\mathrm{R} 1$ & 5971 & 0.97 & 0.81 & 0.72 & 0.37 & 0.28 & 5 & 3 \\
\hline \multirow[t]{3}{*}{$\overline{4}$} & $\overline{\text { Base }}$ & 10454 & $\overline{0.84}$ & 0.75 & 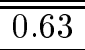 & $\begin{array}{c}-0.13 \\
\end{array}$ & 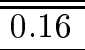 & $\overline{5}$ & 2 \\
\hline & $\mathrm{R} 1$ & 10835 & 0.72 & 0.69 & 0.56 & -0.14 & 0.28 & 5 & 2 \\
\hline & $\mathrm{R} 2$ & 15788 & 0.81 & 0.77 & 0.69 & 0.15 & 0.13 & 5 & 2 \\
\hline \multirow[t]{5}{*}{$\overline{5}$} & Base & 10178 & $\overline{0.94}$ & 0.88 & $\begin{array}{c}0.79 \\
\end{array}$ & $\bar{~} 0.51$ & 0.14 & $\overline{4}$ & 2 \\
\hline & R1 & 4657 & 0.93 & 0.81 & 0.68 & $\begin{array}{l}-0.09 \\
\end{array}$ & 0.32 & 4 & 2 \\
\hline & $\mathrm{R} 2$ & 5616 & 0.94 & 0.81 & 0.79 & -0.47 & 0.06 & 4 & 2 \\
\hline & R3 & 9215 & 0.96 & 0.90 & 0.84 & 0.21 & 0.14 & 4 & 2 \\
\hline & $\mathrm{R} 4$ & 3544 & 0.90 & 0.55 & 0.51 & -1.90 & 0.20 & 4 & 2 \\
\hline \multirow[t]{13}{*}{6} & Base & 5829 & 0.96 & 0.36 & 0.62 & -0.19 & 0 & 1 & 1 \\
\hline & R1 & 2684 & 0.98 & -0.14 & 0.88 & -0.38 & 0 & 1 & 1 \\
\hline & $\mathrm{R} 2$ & 4354 & 0.99 & 0.20 & 0.96 & 0.40 & 0 & 1 & 1 \\
\hline & R3 & 6510 & 0.97 & 0.53 & 1.00 & 0.97 & 0 & 1 & 1 \\
\hline & $\mathrm{R} 4$ & 8688 & 0.92 & 0.63 & 0.87 & 0.41 & 0 & 1 & 1 \\
\hline & R5 & 3079 & 0.80 & 0.38 & 0.81 & 0.32 & 0 & 1 & 1 \\
\hline & R6 & 4384 & 0.96 & 0.66 & 0.78 & 0.47 & $\overline{0}$ & 1 & 1 \\
\hline & R7 & 4047 & 0.92 & 0.53 & 0.82 & 0.27 & 0 & 1 & 1 \\
\hline & R8 & 3935 & 0.89 & 0.57 & 0.80 & 0.53 & 0 & 1 & 1 \\
\hline & R9 & 3201 & 0.90 & 0.55 & 0.85 & 0.61 & 0 & 1 & 1 \\
\hline & R10 & 3895 & 0.91 & 0.61 & 0.88 & 0.26 & 0 & 1 & 1 \\
\hline & R11 & 5878 & 0.94 & 0.65 & 0.67 & 0.22 & 0 & 1 & 1 \\
\hline & R12 & 7421 & 0.86 & 0.63 & 0.51 & 0.21 & 0 & 1 & 1 \\
\hline$\overline{7}$ & Base & 1018 & $\overline{0.81}$ & 0.50 & 0.86 & 0.13 & 0 & 1 & 1 \\
\hline$\overline{88}$ & $\overline{\text { Base }}$ & $\overline{44521}$ & $\overline{c 0.99}$ & $\bar{~} 0.92$ & 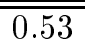 & "0.30 & $\overline{0}$ & $\overline{1}$ & $\overline{1}$ \\
\hline
\end{tabular}


Table 1..6: Knowledge-Based Tumor vs. kNN. (Pat. = Patient; GT $=$ Ground truth volume; $\mathrm{KB}=$ Knowledge-based; $\mathrm{kNN}$ SDP $=\mathrm{kNN}$ Standard Deviation as Percentage of Tumor Volume; Manual $\mathrm{kNN}=\mathrm{kNN}$ volume after manual tumor extraction.)

\begin{tabular}{|c|c|c|c|c|c|c|}
\hline Pat. & Scan & $\begin{array}{c}\text { GT } \\
\text { Vol. }\end{array}$ & $\begin{array}{c}\text { KB } \\
\text { Vol. }\end{array}$ & $\begin{array}{c}\text { kNN } \\
\text { Vol. }\end{array}$ & $\begin{array}{c}\text { kNN } \\
\text { SDP }\end{array}$ & $\begin{array}{c}\text { Manual } \\
\text { kNN }\end{array}$ \\
\hline \hline 1 & Base & 7213 & 8561 & 10022 & 0.07 & 6334 \\
\hline & R1 & 7240 & 8829 & 11958 & 0.19 & 6794 \\
\hline & R2 & 7470 & 9928 & 11576 & 0.14 & 6616 \\
\hline & R3 & 6395 & 8132 & 10870 & 0.40 & 5901 \\
\hline & R4 & 6560 & 8614 & 12115 & 0.07 & 5690 \\
\hline \hline 5 & Base & 10178 & 10784 & 14044 & 0.14 & 7938 \\
\hline & R1 & 4657 & 5489 & 10279 & 0.32 & 2834 \\
\hline & R2 & 5616 & 6773 & 18603 & 0.06 & 3952 \\
\hline & R3 & 9215 & 9833 & 18210 & 0.14 & 6729 \\
\hline & R4 & 3544 & 5730 & 19138 & 0.20 & 3035 \\
\hline
\end{tabular}

Table A., manually extracted kNN consistently underestimated tumor volume, meaning the method missed more radiologist verified tumor than the knowledge-based method, significantly in some cases.

A comparison is also made against the semi-supervised FCM (ssFCM) algorithm, which was initialized with the same ROI's used to initialize $\mathrm{kNN}$ in Table $1 . .5[52,58,53]$. The resultant ssFCM segmentation was then used to initialize ISG, a commercially available seed-growing tool (ISG Technologies, Toronto, Canada) for supervised evaluation of tumor volumes. The ISG processing also removed any extra-cranial tissues found in the ssFCM segmentation. Results available for the volumes processed by ssFCM and ISG are shown in Table 1..7. The results reported in Table $1 . .7$ are a mean over the set of trials performed for that volume and thus have a standard deviation, also listed. Only final tumor volumes were available from $[52,58,53] \mathrm{ssFCM}$ and ISG.

In terms of absolute difference, the ssFCM approach was closer to ground truth than the knowledge-based method in 10 out of 18 volumes, with 6 of these cases by more than the standard deviation of the ssFCM volume. In the 8 cases where the knowledge-based method gave better results, however, 7 of them were better than ssFCM by more than the standard deviation. The knowledge-based method performs better against ISG, in 9 out of 16 cases, with all 9 by more than the standard deviation of ISG. Furthermore, ssFCM underestimated total tumor volume in 12 instances, while ISG underestimated tumor volume in all 16 available volumes, which is not helpful for any use involving treatment since the methods missed more radiologist verified tumor.

\section{B. Evaluation Over Repeat Scans}

An important use of tumor volume estimation is in tracking a tumor's response to treatment based on its growth/shrinkage. From the 33 volumes available, 25 "transitions" could be tracked (e.g., Baseline Scan to Repeat Scan 1 is one transition). Examining tumor growth/shrinkage over repeat scans, the knowledge-based method failed to properly track 3 of 25 transitions (12\%). The kNN method, without manual tumor extraction, failed on 8 of 25 transitions (32\%), while the manually extracted kNN volumes failed in 2 of 10 transitions (20\%). The ssFCM method failed on 3 of 13 transitions (23\%), while ISG failed on 4 out of $12(33 \%)$. Since the $\mathrm{kNN}$, ssFCM, and ISG volumes are based on multiple trials, it is difficult to assign a specific cause, although the im- 
Table 1..7: Knowledge-Based Tumor vs. ssFCM and ISG. Results for Patient 6, 7, and 8 for ssFCM and ISG were unavailable. (Pat. = Patient; GT Vol. = Ground Truth Volume; KB = Knowledge-based; SDP = Standard Deviation as Percentage of Tumor Volume; \# Trial $=$ Number of Trials; \# Obs. = Number of Observers; N/A = Not Available.)

\begin{tabular}{|c|c|c|c|c|c|c|c|c|c|}
\hline Pat. & Scan & $\begin{array}{c}\text { GT } \\
\text { Vol. }\end{array}$ & $\begin{array}{c}\text { KB } \\
\text { Vol. }\end{array}$ & $\begin{array}{c}\text { ssFCM } \\
\text { Vol. }\end{array}$ & $\begin{array}{c}\text { ssFCM } \\
\text { SD }\end{array}$ & $\begin{array}{c}\text { ISG } \\
\text { Vol. }\end{array}$ & $\begin{array}{c}\text { ISG } \\
\text { SD }\end{array}$ & $\begin{array}{c}\# \\
\text { Trials }\end{array}$ & $\begin{array}{c}\# \\
\text { Obs. }\end{array}$ \\
\hline \hline 1 & Base & 7213 & 8561 & 8015 & 0.07 & 6067 & 0.05 & 5 & 2 \\
\hline & R1 & 7240 & 8829 & 7757 & 0.18 & 5956 & 0.03 & 5 & 2 \\
\hline & R2 & 7470 & 9928 & 7362 & 0.03 & 6087 & 0.03 & 5 & 2 \\
\hline & R3 & 6395 & 8132 & 7185 & 0.09 & 5361 & 0.02 & 5 & 2 \\
\hline \hline & R4 & 6560 & 8614 & 7332 & 0.19 & 5172 & 0.04 & 5 & 2 \\
\hline \hline & Base & 12230 & 15494 & 12457 & 0.06 & 10027 & 0.06 & 5 & 2 \\
\hline & R1 & 14609 & 12874 & 10916 & 0.13 & 7120 & 0.15 & 5 & 2 \\
\hline \hline & R2 & 20924 & 19541 & 13498 & 0.06 & 10120 & 0.06 & 5 & 2 \\
\hline \hline & Base & 10892 & 10855 & 8018 & 0.08 & N/A & N/A & 5 & 3 \\
\hline & R1 & 5971 & 7688 & 5337 & 0.03 & N/A & N/A & 5 & 3 \\
\hline & Base & 10454 & 10517 & 8563 & 0.05 & 6258 & 0.02 & 4 & 2 \\
\hline \hline & R1 & 10835 & 8614 & 8901 & 0.04 & 6040 & 0.03 & 4 & 2 \\
\hline 5 & R2 & 15788 & 15045 & 14080 & 0.06 & 10819 & 0.17 & 4 & 2 \\
\hline & Rase & 10178 & 10784 & 10334 & 0.06 & 7562 & 0.09 & 4 & 2 \\
\hline & R2 & 5657 & 5489 & 3813 & 0.15 & 3142 & 0.24 & 4 & 2 \\
\hline & R3 & 9215 & 9833 & 7852 & 0.02 & 7300 & 0.06 & 4 & 2 \\
\hline & R4 & 3544 & 5730 & 3110 & 0.19 & 2311 & 0.13 & 4 & 2 \\
\hline
\end{tabular}




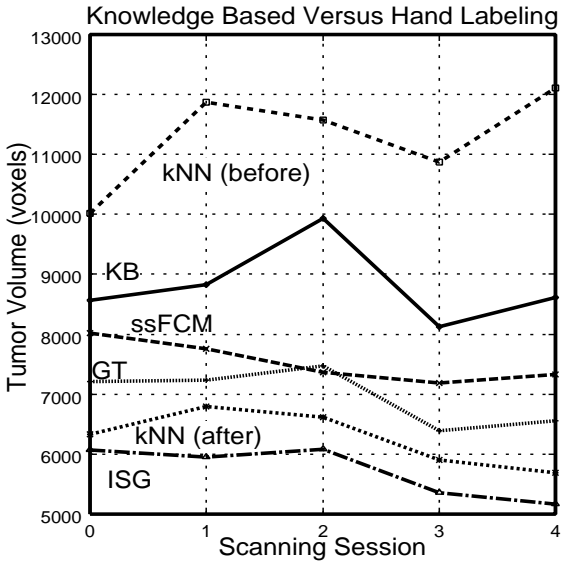

(a) Patient 1

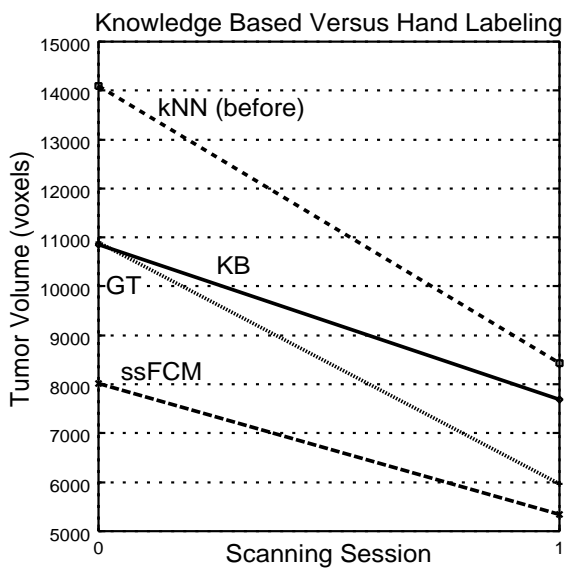

(c) Patient 3

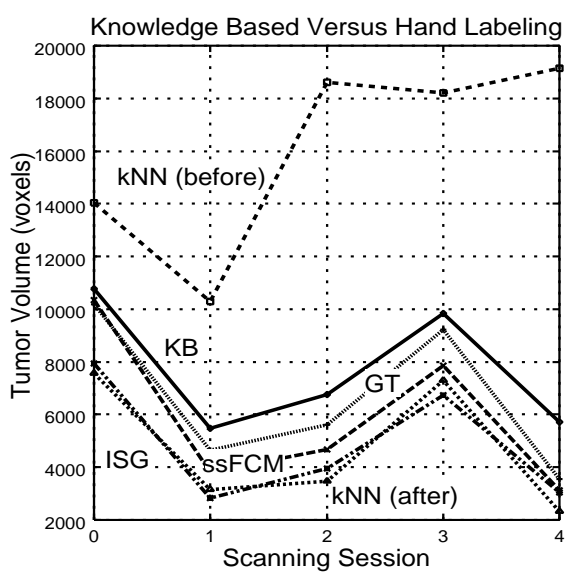

(e) Patient 5

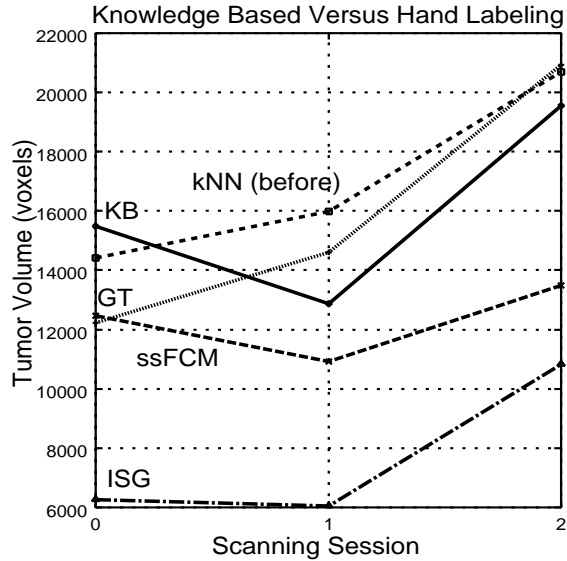

(b) Patient 2

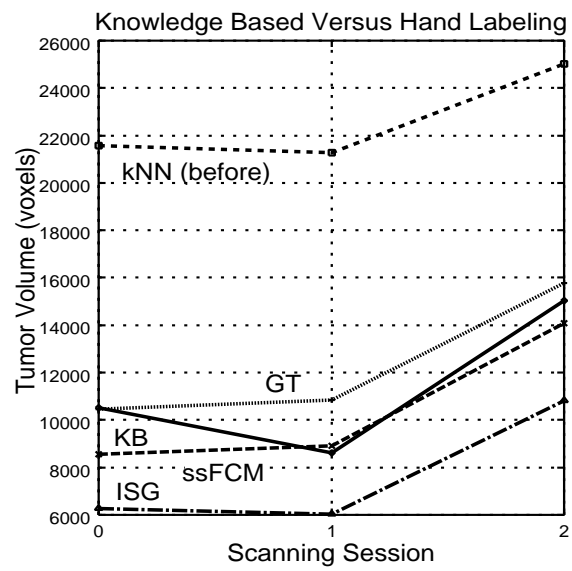

(d) Patient 4

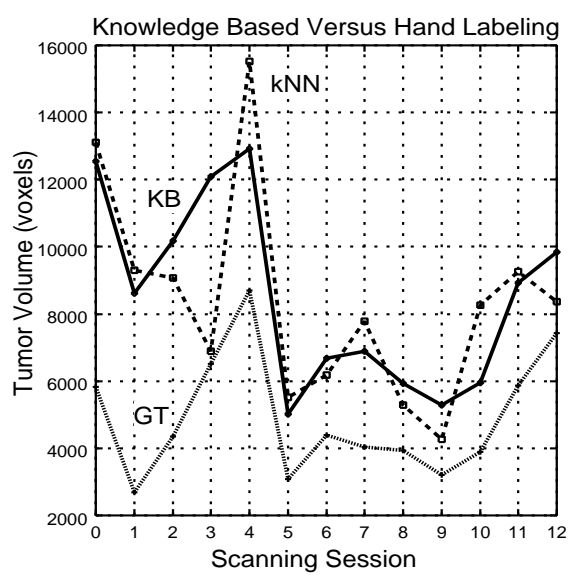

(f) Patient 6

Figure 1.14: Tracking Tumor Growth/Shrinkage Over Repeat Scans. KB=KnowledgeBased System. GT=Ground Truth. 


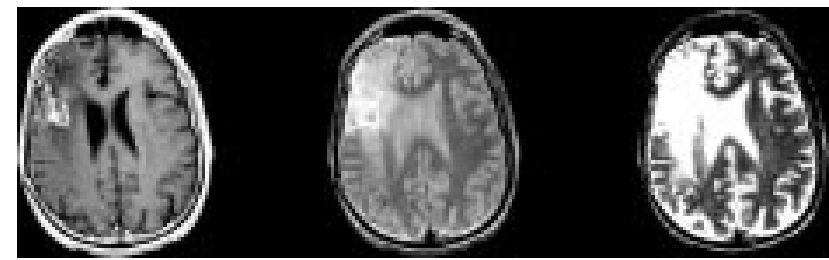

(a) Raw Image

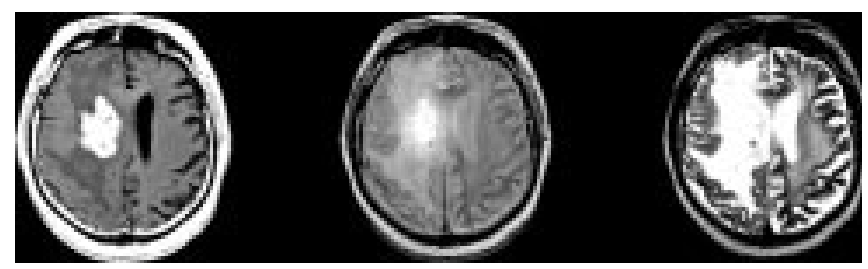

(d) Raw Image

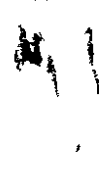

(b) KB Tumor

(c) GT Tumor

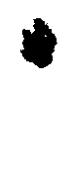

(e) KB Tumor (f) GT Tumor

Figure 1.15: Comparison of Knowledge-Based Tumor Segmentation Vs. Ground Truth. Worst case (a-c) and best case (d-f).

portance of supervised removal of extra-cranial tissues, handled automatically by the knowledge-based system, should be noted. Also, as a percentage, the knowledge-based system had a lower rate of failure than the supervised methods.

For the knowledge-based system, the failure in Patient 6, Repeat Scans 8 to 9, was due to slight over-estimation of tumor volume in Scan 9 where the T1 threshold was set too low. The failure of Patient 4, Baseline to Repeat Scan 1 was due primarily to poor extraction of the intra-cranial region in 3 slices, resulting in significant tumor loss. The third transition failure, Patient 2: Baseline to Repeat Scan 1, was due primarily to the presence of a significant amount of fluid, possibly hemorrhage, in the Baseline scan. This distorted the PD histogram used in Stage Two, resulting in significant overestimation. In Repeat Scan 1, however, not only had the fluid disappeared, but pathology reports noted the slight decrease in gadolinium enhancement. The tumor boundaries were not properly captured in Stage Five and resulted in a T1 threshold that was too high. Thus, the initial overestimation followed by the decreased gadolinium enhancement caused the trend to appear to be tumor shrinkage instead of growth. Figure 1.14 illustrates the total tumor volumes for the six patients with repeat scans using knowledge-based, kNN, and radiologist-verified ground truth.

Examples of knowledge-based segmentation versus ground-truth are shown in Figure 1.15 to visually show the knowledge-based system tumor correspondence to radiologistlabeled tumor. Figures 1.15(a-c) show a worst case segmentation, while (d-f) show a best case. Both examples are from slices in the test set.

\section{Discussion}

We have described a knowledge-based multi-spectral analysis tool that segments and labels glioblastoma-multiforme tumor. The guidance of the knowledge base gives this system additional power and flexibility by allowing unsupervised segmentation and classification decisions to be made through iterative/successive refinement. This is in contrast to most other multi-spectral efforts such as [51, 54, 19] which attempt to segment the entire brain image in one step, based on either statistical or (un)supervised classification methods. 
The knowledge base was initially built with a general set of heuristics comparing the effects of different pulse sequences on different types of tissues. This process is called "knowledge-engineering" as we had to decide which knowledge was most useful for the goal of tumor segmentation, followed by the process of implementing such information into a rule-based system. More importantly, the training set used was quite small seventeen slices over three patients. Yet, the system performed well. A larger training set would most likely allow new and more effective trends and characteristics to be revealed. Thresholds used to handle a certain subset of the training set could be better generalized.

The slices processed had a relatively large thickness of $5 \mathrm{~mm}$. Thinner slices which exhibit a reduced partial-volume effect and allow better tissue contrast could be used. While relying on feature space distributions, the system was developed using general tissue characteristics and relative relationships between tissues to avoid dependence upon specific feature-domain values. The particular slices were acquired with the same parameters, but gadolinium-enhancement has been found to be generally very robust in different protocols and thickness [4, 24]. Should acquisition parameter dependence become an issue, given a large enough training base across multiple parameters, the knowledge base could automatically adjust to a slice's specific parameters since such information is easily included when processing starts. The patient volumes processed had received various degrees of treatment, including surgery, radiation and chemo-therapy both before and between scans. Yet, despite the changes these treatments can cause, such as demyelinization of white matter, no modifications to the knowledge based system were necessary. Other approaches, like neural networks [1] or any sort of supervised method which is based on a specific set of training examples could have difficulties in dealing with slightly different imaging protocols and the effects of treatment.

The promise of the knowledge-based system as a useful tool is demonstrated by the successful performance of the system on the processed slices. The final KB segmentations compare well with radiologist-labeled "ground truth" images. The knowledgebased system also compared well with supervised methods, and was able to segment tumor without the need for (multiple) human-based ROI's or post-processing, which make supervised methods clinically impractical.

As stated in the introduction, no method of quantitating tumor volumes is widely accepted and used in clinical practice [38]. An method by the Eastern Cooperative Oncology group [17] approximates tumor area in the single MR slice with the largest contiguous, well-defined tumor evident. The longest tumor diameter is multiplied by its perpendicular to yield an area. Changes greater than $25 \%$ in the area of a tumor over time are used, in conjunction with visual observations, to classify tumor response to treatment into five categories from complete response (no measurable tumor left) to progression. This approach does not address full tumor volume, depends on the exact boundary choices, and the shape of the tumor [31, 17]. By itself, the approach can lead to inaccurate growth/shrinkage decisions [10].

Overall, the knowledge-based approach tended to overestimate the tumor volume. Only two patients (2 and 4 ) showed noticeable underestimation by the knowledge-based system. In all other cases, the knowledge-based system shows a number of "false positives." Since the system segments tumor by removing only pixels proven not to be tumor, leaving anything that remains being labeled as tumor, a higher level of false positives is not inconsistent with the paradigm. The final $\mathrm{T} 1$ threshold is applied using a fixed parameter $\sigma$, but the knowledge-based system's errors suggest that creating rules to automatically set $\sigma$ (making Stage Five more adaptive to tumors with different degrees of enhancement) is worthy of investigation.

It should also be noted, however, that the process of creating ground truth images 
is very imprecise [40] and has approximately a $5 \%$ inter-observer variability in tumor volume [56]. All brain tumors have micro-infiltration beyond the borders defined with gadolinium enhancement. This is especially true in glioblastoma-multiformes, which are the most aggressive grade of primary glioma brain tumors, and no one can tell the exact tumor borders, even with invasive histopathological methods $[9,18,41]$, which were unavailable. Ground truth images mark the areas of tumor exhibiting the most angiogenesis (formation of blood vessels, resulting in the greatest gadolinium concentration) and represent those pixels which are "statistically most likely" to contain tumor $[40,41]$. Such pixels would have the highest level of agreement agreement between radiologists, but they do not guarantee that all tumor has been identified [40]. Therefore, the knowledge-based system may often capture tumor boundaries that extend into areas showing lower degrees of angiogenesis (which would still be treated during therapy) [41].

Future work includes addressing the problems noted in Section IV. to improve the system's performance. The high number of false positives, which appear to be a matter of tumor boundaries, can be reduced by applying a final threshold in T1-space (the feature image used primarily by radiologists in determining final tumor boundaries). Our primary concern was losing as little ground truth tumor as possible. Expanding the training set to include more patients should expand the generalizability of the knowledge base. The next expected development in this system is to expand the processing range to all slices that intersect the brain cerebrum. Introducing new tumor types, such as lower grade gliomas will also be considered, as will complete labeling of all remaining tissues. Also, newer MRI systems may provide additional features, such as diffusion images or edge strength to estimate tumor boundaries, which can be readily included into the knowledge base. The knowledge-base also allows straightforward expansion as new tools are found effective.

In conclusion, the knowledge-based system is a multi-spectral tool that shows promise in effectively segmenting glioblastoma-multiforme tumors without the need for human supervision. It has the potential of being a useful tool for segmenting tumor for therapy planning, and tracking tumor response. Lastly, the knowledge-based paradigm allows easy integration of new domain information and processing tools into the existing system when other types of pathology and MR data are considered.

\section{Acknowledgements}

This research was partially supported by a grant from the Whitaker foundation and a grant from the National Cancer Institute (CA59 425-01). Thanks to Dr. Mohan Vaidyanathan for his assistance in the ground truth work.

\section{References}

[1] Amartur, S., Piriano, D., and Takefuji, Y. Optimization neural networks for the segmentation of magnetic resonance images. IEEE TMI 11, 2 (June 1992), 215-221.

[2] Bomans, M., Höhne, K., Tiede, U., and Riemer, M. 3D segmentation of MR images of the head for 3D display. IEEE TMI 9 (1990), 177-183.

[3] Bottomley, P., Foster, T., Argersinger, R., and Pfeiffer, L. A review of normal tissue hydrogen NMR relaxation times and relaxation mechanisms from 1-100 MHz: Dependency on tissue type, NMR frequency, temperature, species, excision and age. Medical Physics 11 (1984), 425-448. 
[4] Bronen, R., and Sze, G. Magnetic resonance imaging contrast agents: Theory and application to the central nervous system. Journal of Neurosurgery 73 (1990), 820-839.

[5] Cannon, R., Dave, J., and Bezdek, J. Efficient implementation of the fuzzy c-mean clustering algorithms. IEEE Transactions on Pattern Analysis and Machine Intelligence 8, 2 (1986), 248-255.

[6] Clark, M., Hall, L., Goldgof, D., and et al. MRI segmentation using fuzzy clustering techniques: Integrating knowledge. IEEE Engineering in Medicine and Biology 13, 5 (1994), 730-742.

[7] Clark, M., Hall, L., Li, C., and Goldgof, D. Knowledge based (re-)clustering. In Proceedings of the 12th IAPR International Conference on Pattern Recognition (1994), pp. 245-250. Jerusalem, Israel.

[8] Clark, M. C. Knowledge-Guided Processing of Magnetic Resonance Images of the Brain. PhD thesis, University of South Florida, 1997.

[9] Clarke, L., Velthuizen, R., Camacho, M., Heine, J., Vaydianathan, M., Hall, L., Thatcher, R., and Silbiger, M. MRI segmentation: Methods and applications. Magnetic Resonance Imaging 13, 3 (1995), 343-368.

[10] Clarke, L., Velthuizen, R., Clark, M., Gaviria, G., Hall, L., Goldgof, D., and et al. MRI measurement of brain tumor response: Comparison of visual metric and automatic segmentation. Submitted to Magnetic Resonance Imaging, June 1997.

[11] Dasarthy, B. Nearest Neighbor (NN) Norms: NN Pattern Classification Techniques. IEEE Computer Society Press, Los Alamitos, Ca., 1991.

[12] Dellepiane, S. Image segmentation: Errors, sensitivity, and uncertainty. In Proceedings of the 13th IEEE EMB Society (1991), vol. 13, pp. 253-254.

[13] Dellepiane, S., Venturi, G., and Vernazza, G. A fuzzy model for the processing and recognition of MR pathological images. In IPMI 1991 (1991), pp. 444-457.

[14] Dougherty, S. Discussions held with Sean Dougherty concerning edge detection algorithms in MR images, September 1997.

[15] Farrar, T. C. An Introduction to Pulse NMR Spectroscopy. Farragut Press, 1987.

[16] Feldman, G. B. Brain tumor facts and figures. The Brain Tumor Society http://www.thts.org/btfacts.htm, July 31997.

[17] Feun, L. Double-blind randomized trial of the anti-progestational agent mifepristone in the treatment of unresectable meningioma, phase iii. Tech. Rep. SWOG9005, University of South Florida, Tampa, Fl., Southwest Oncology Group, 1995.

[18] Galloway, R., Maciunas, R., and Failinger, A. Factors affecting perceived tumor volumes in magnetic resonance imaging. Annals of Biomedical Engineering 21 (1993), 367-375.

[19] Gerig, G., Martin, J., Kikinis, R., and et al. Automating segmentation of dual-echo MR head data. In The 12th International Conference of Information Processing in Medical Imaging (IPMI 1991) (1991).

[20] Giarratano, J., and Riley, G. Expert Systems: Principles and Programming, second ed. Boston: PWS Publishing, 1994.

[21] Gibbs, P., Buckley, D., Blackband, S., and Horsman, A. Tumor volume determination from MR images by morphological segmentation. Physics in Medicine and Biology 41, 11 (November 1996), 2437-2446.

[22] Gong, L., and Kulikowski, C. Automatic segmentation of brain images: Selection of region extraction methods. In SPIE Vol. 1450 Biomedical Image Processing II (1991), SPIE, pp. 144-153. 
[23] Hall, L., Bensaid, A., Clarke, L., and et al. A comparison of neural network and fuzzy clustering techniques in segmenting magnetic resonance images of the brain. IEEE Transactions on Neural Networks 3, 5 (1992), 672-682.

[24] Hendrick, R., and Haacke, E. Basic physics of MR contrast agents and maximization of image contrast. JMRI 3, 1 (1993), 137-148.

[25] Hillman, G., Chang, C., Ying, H., and et al. Automatic system for brain MRI analysis using a novel combination of fuzzy rule-based and automatic clustering techniques. In Medical Imaging 1995: Image Processing (February 1995), SPIE, pp. 16-25. San Diego, CA.

[26] Jain, A. Fundamentals of Digital Image Processing. Englewood Cliffs,NJ: Prentice Hall, 1989.

[27] Jain, R., Kasturi, R., and Schunck, B. Machine Vision. McGraw-Hill, Inc., 1995.

[28] Kamber, M., Shingal, R., Collins, D., Francis, G., and Evans, A. Modelbased 3D segmentation of multiple sclerosis lesions in magnetic resonance brain images. IEEE TMI 14, 3 (1995), 442-453.

[29] Kikinis, R., Shenton, M., Gerig, G., and et al. Routine quantitative analysis of brain and cerebrospinal fluid spaces with MR imaging. JMRI 2 (1992), 619-629.

[30] Kischell, E., Kehtarnavaz, N., Hillman, G., Levin, H., Lilly, M., and Kent, T. Classification of brain compartments and head injury lesions by neural networks applied to MRI. Neuroradiology 37 (1995), 535-541.

[31] Laperrire, N., and Berstein, M. Radiotherapy for brain tumors. CA - A Cancer Journal for Clinicians \& (1994), 96-108.

[32] Leeds, N., and Jackson, E. Current imaging techniques for the evaluation of brain neoplasms. Current Science 6 (1994), 254-261.

[33] Li, C. Knowledge based classification and tissue labeling of magnetic resonance images of the brain. Master's thesis, University of South Florida, 1993.

[34] Li, C., Goldgof, D., and Hall, L. Automatic segmentation and tissue labeling of MR brain images. IEEE TMI 12, 4 (December 1993), 740-750.

[35] Li, X., Bhide, S., and Kabuka, M. Labeling of MR brain images using boolean neural network. IEEE TMI 15, 2 (1996), 628-638.

[36] Lufkin, R. B. The MRI Manual. Year Book Medical Publishers, Inc., 1990.

[37] Menhardt, W., and Schmidt, K. Computer vision on magnetic resonance images. Pattern Recognition Letters 8, 2 (September 1988), 73-85.

[38] Murtagh, R., Phuphanich, S., Imam, N., Clarke, L., Vaidyanathan, M., and et.al. Novel methods of evaluating the growth response patterns of treated brain tumors. Cancer Control (1995), 293-299.

[39] Murtaugh, F. Discussions held with Dr. F. Reed Murtaugh, M.D., Dept. of Radiology, University of South Florida, April 1997.

[40] Murtaugh, F. Discussions held with Dr. F. Reed Murtaugh, M.D., Dept. of Radiology, University of South Florida, October, 11997.

[41] Murtaugh, F. Discussions held with Dr. F. Reed Murtaugh, M.D., Dept. of Radiology, University of South Florida, October, 221997.

[42] Namasivayam, A., and Hall, L. Integrating fuzzy rules into the fast, robust segmentation of magnetic resonance images. In New Frontiers in Fuzzy Logic and Soft Computing Biennial Conference of the North American Fuzzy Information Processing Society - NAFIPS 1996 (1996), pp. 23-27. Piscataway, NJ.

[43] Novelline, R., and Squire, L. Living Anatomy. Hanley and Belfus, 1987. 
[44] Özkan, M., Dawant, B., and Maciunas, R. Neural-network-based segmentation of multi-modal medical images: A comparative and prospective study. IEEE TMI 12, 3 (September 1993), 534-545.

[45] Raff, U., and Newman, F. Automated lesion detection and lesion quantitation in MR images using autoassociative memory. Medical Physics 19 (1992), 71-77.

[46] Riley, G. Version 4.3 CLIPS reference manual. Tech. Rep. JSC-22948, Artificial Intelligence Section, Lyndon B. Johnson Space Center, 1989.

[47] Schnitzlein, H., and Murtaugh, F. R.Imaging Anatomy of the Head and Spine: A Photographic Color Atlas of MRI, CT, Gross, and Microscopic Anatomy in Axial, Coronal, and Sagittal Planes, second ed. Baltimore: Urban \& Schwarzenberg, 1990.

[48] Stark, D. D., and William G. Bradley, J. Magnetic Resonance Imaging, Second Ed., Volume One. Mosby Year Book, 1992.

[49] Tao, C., and Thompson, W. A fuzzy if-then approach to edge detection. In 1993 IEEE International Conference on Fuzzy Systems (1993), IEEE, pp. 1356-1360.

[50] Taxt, T., and Lundervold, A. Multispectral analysis of the brain in magnetic resonance imaging. In IEEE Workshop on Biomedical Image Analysis (1994), pp. 33-42. Los Alamitos, CA, USA.

[51] Taxt, T., and Lundervold, A.Multispectral analysis of the brain using magnetic resonance imaging. IEEE TMI 13, 3 (September 1994), 470-481.

[52] Vaidyanathan, M., Velthuizen, R., Clarke, L., and Hall, L. Quantitation of brain tumor in MRI for treatment planning. In Proceedings of the 16th Annual International Conference of the IEEE Engineering in Medicine and Biology Society (1994), vol. 16, pp. 555-556.

[53] Vaidyanathan, M., Velthuizen, R., Venugopal, P., and Clarke, L. Tumor volume measurements using supervised and semi-supervised MRI segmentation methods. In Artificial Neural Networks in Engineering - Proceedings (ANNIE 1994) (1994), vol. 4, pp. 629-637.

[54] Vannier, M., Butterfield, R., Jordan, D., and et al. Multispectral analysis of magnetic resonance images. Radiology 154, 1 (January 1985), 221-224.

[55] Vannier, M., Speidel, C., and Rickmans, D. Magnetic resonance imaging multispectral tissue classification. News Physiol Sci 3 (August 1988), 148-154.

[56] Velthuizen, R., and Clarke, L. An interface for validation of MR image segmentations. In Proceedings of the 16th Annual International Conference of the IEEE Engineering in Medicine and Biology Society (1994), pp. 547-548.

[57] Velthuizen, R., Hall, L., and Clarke, L. Unsupervised fuzzy segmentation of 3D magnetic resonance brain images. In Proceedings of the ISET SPIE 1993 International Symposium on Electronic Images: Science \& Technology (1993), vol. 1905, pp. 627-635. San Jose, CA, Jan 31-Feb 4.

[58] Velthuizen, R., Phuphanich, S., Clarke, L., Hall, L., and et al.Unsupervised tumor volume measurement using magnetic resonance brain images. JMRI 5, 5 (1995), 594-605.

[59] Wu, Z., and Leahy, R. A graph theoretic approach to segmentation of MR images. In SPIE Vol.1450 Biomedical Image Processing II (1991), pp. 120-132.

[60] Wu, Z., and Leahy, R. Image segmentation via edge contour finding: A graph theoretic approach. In IEEE Computer Vision and Pattern Recognition (1992), pp. 613-619. 